Published in final edited form as:

Med Image Anal. 2014 July ; 18(5): 795-807. doi:10.1016/j.media.2014.04.005.

\title{
Characterization of U-shape Streamline Fibers: Methods and Applications
}

\author{
Tuo Zhang ${ }^{2,1,{ }^{*}, \text { Hanbo Chen }}{ }^{1,{ }^{*}}$, Lei Guo ${ }^{2}$, Kaiming $\mathrm{Li}^{3}$, Longchuan $\mathrm{Li}^{3}$, Shu Zhang ${ }^{1}$, \\ Dinggang Shen ${ }^{4}$, Xiaoping $\mathrm{Hu}^{3,{ }^{* *}}$, and Tianming $\mathrm{Liu}^{1,{ }^{1 *}}$ \\ ${ }^{1}$ Department of Computer Science and Bioimaging Research Center, The University of Georgia, \\ Athens, GA \\ ${ }^{2}$ School of Automation, Northwestern Polytechnic University, China \\ ${ }^{3}$ Biomedical Imaging Technology Center, Emory University, Atlanta, GA \\ ${ }^{4}$ Department of Radiology and Biomedical Research Imaging Center, UNC Chapel Hill, NC
}

\begin{abstract}
Diffusion tensor imaging (DTI), high angular resolution diffusion imaging (HARDI), and Diffusion Spectrum Imaging (DSI) have been widely used in the neuroimaging field to examine the macro-scale fiber connection patterns in the cerebral cortex. However, the topographic and geometric relationships between diffusion imaging derived streamline fiber connection patterns and cortical folding patterns remain largely unknown. This paper specifically identifies and characterizes the U-shapes of diffusion imaging derived streamline fibers via a novel fiber clustering framework and examines their co-localization patterns with cortical sulci based on DTI, HARDI, and DSI datasets of human, chimpanzee and macaque brains. We verified the presence of these U-shaped streamline fibers that connect neighboring gyri by coursing around cortical sulci such as the central sulcus, pre-central sulcus, post-central sulcus, superior temporal sulcus, inferior frontal sulcus, and intra-parietal sulcus. This study also verified the existence of U-shape fibers across data modalities (DTI/HARDI/DSI) and primate species (macaque, chimpanzee and human), and suggests that the common pattern of U-shape fibers coursing around sulci is evolutionarilypreserved in cortical architectures.
\end{abstract}

\section{Keywords}

DSI; HARDI; DTI; fiber shapes; shape analysis

\footnotetext{
(C) 2014 Elsevier B.V. All rights reserved.

${ }_{*}^{* *}$ Joint correspondence authors.

* These authors contributed equally to this work.

Publisher's Disclaimer: This is a PDF file of an unedited manuscript that has been accepted for publication. As a service to our customers we are providing this early version of the manuscript. The manuscript will undergo copyediting, typesetting, and review of the resulting proof before it is published in its final citable form. Please note that during the production process errors may be discovered which could affect the content, and all legal disclaimers that apply to the journal pertain.
} 


\section{Introduction}

Folding is an essential shape characteristic of the cerebral cortex in primate brains such as macaque, chimpanzee and human. The convex and concave cortical structures of gyri and sulci emerge from complex cortical folding processes during neurodevelopment (Richman et al., 1975; Rakic, 1988; Welker, 1990; Sur and Rubenstein, 2005; Rash and Grove, 2006;

Dennis et al., 2007). Meanwhile, large-scale axonal wiring is another key determinant that sculpts the cerebral cortex into a functional working system during neurodevelopment (O'Leary, 1989; Brown et al., 2002; Passingham, et al., 2002; Berghuis et al., 2007; Chédotal and Richards, 2010). In the past few decades, the neuroscientific communities have made remarkable progresses in understanding these two prominent structural attributes of the primate cortices (Rakic, 1988; Sur and Rubenstein, 2005; Chédotal and Richards, 2010). However, the intrinsic relationship between these two general cross-species attributes, as well as the underlying organizational principles, that construct the structural and functional architecture of the cerebral cortex, remains largely unknown.

Thanks to recent advancements of modern in-vivo brain imaging techniques, in particular, diffusion spectrum imaging (DSI) (e.g., Wedeen et al., 2012), high angular resolution diffusion imaging (HARDI) (e.g., Tuch et al., 2002) and diffusion tensor imaging (DTI) (e.g., Basser and Pierpaoli 1996; Mori 2006, we are now able to quantitatively measure the brain's in-vivo fiber wiring diagrams with decent spatial resolutions. Despite some limitations in data acquisition and processing algorithms, the diffusion MRI data has shown consistence with known pathways of major fiber tracts in the brain (Conturo et al, 1999, LeBihan, 2003, Catani et al, 2003

Particularly, the joint representation and modeling approach of brain structure and connection patterns has demonstrated its advantages in elucidating the structural brain architectures and possible functional mechanisms (e.g., Behrens et al., 2003; Rilling et al., 2008; Zhu et al., 2011). Recently, our analyses of a number of primate/human brains based on the joint representation of cortical gyral folding and DTI-derived fiber connection patterns (Nie et al., 2011) showed that the streamline fibers connected to gyri are denser, than those connected to sulci in human, chimpanzee and macaque brains. This finding is illustrated in Figure 1(a), which is a joint representation of a sagittal section through the middle part of human brain. As can be seen, DTI-derived fiber trajectories' terminations (yellow arrows) concentrate on gyral regions highlighted by the blue arrows. In a recent follow-up work (Chen et al., 2012), our quantitative analyses of DTI and MRI data based on the same joint representation consistently demonstrated that the structural fiber connection pattern closely follows the gyral folding pattern in the tangent direction to the cortical sphere, and this close relationship is well-preserved in the neocortices of macaque, chimpanzee and human being, despite the progressively increasing complexity and variability of cortical folding and structural connection patterns in these species. This finding is illustrated by the blue arrows in Figure 1(b), from which we can observe that the convex cortical gyral shapes are connected by dense fibers orienting along the tangent directions of the gyral crest lines. In this figure, we mapped the orientations of the fiber terminations onto the cortical surface patch. Red/blue colors indicate that the fiber 
termination apparently orients left/right (from the view angle of Figure 1(b)). Green color indicates the fiber termination orient vertically to the tangent direction.

Despite the above findings regarding the relationships between axonal wiring and gyral folding patterns, however, the topographic and geometric relationships between axonal fiber connection patterns and cortical sulci remain largely unknown. Here, our extensive observations revealed that the co-localization patterns of fibers of different shapes with sulcal regions can be categorized into at least two basic types. Figure 1 shows two examples of different co-localization patterns. Figure 1(a) illustrates one example of how straight line shape fiber bundles with different orientations course around the sulcal regions (highlighted by black arrows) by forming rectilinear crossings beneath sulci. Figure 1(c) shows examples of U-shape fiber bundles which course around the sulci by embracing them and the terminations (yellow arrows) of those bundles concentrate on the neighboring gyri. In this work, we particularly focus on characterization of U-shape fibers (Figure 1(c)). Although Ushape fibers have been widely reported in literature (e.g., Wedeen, V.J., 2012, Van Essen D.C., 1997), we developed a new analysis pipeline which starts from U-shape identification and ends up with several result interpretations like co-localization patterns of $\mathrm{U}$-shape fibers with cortical sulci, U-shape fiber wiring diagram and ratios.

More specifically, in order to identify U-shape fibers, we developed a novel fiber clustering framework based on the one in Hu et al., 2010. Compared with the method proposed in other studies on fiber shape analysis and clustering (e.g., Corouge et al., 2004, Gerig et al., 2004, O'Donnell et al., 2006), the reason we adopted the methods in Hu et al., 2010 lies in that it presented a feature set to describe each single fiber and are promising to be applied to large scale dataset, which is very suitable for this study, because our goal is comparing the fiber shape across species and across datasets (referred by us as 'large scale') and there will be millions of fiber tracts to deal with. However, the original methods in Hu et al., 2010 still have limitations when applied on the large scale and cross-species datasets in this paper. For example, considering the computation load when dealing with such a huge dataset in this paper, one possible approach could be clustering fiber tracts of each species via the method in Hu et al. separately. However, the problem is that the clustering results could be possibly biased by species variation. As a result, the AP algorithm cannot be directly applied on such huge and cross-species datasets. Therefore, while adopting the similar fiber features as those in $\mathrm{Hu}$, et al., 2010, we substantially improved the computational framework in $\mathrm{Hu}$, et al., 2010 into a two-step clustering framework. That is, firstly, we sample 6000 fibers tracts from all datasets and apply the AP directly to obtain the cluster centers. Secondly, we use the k-means clustering method to propagate the clustering results onto all datasets by using the cluster centers as initializations. Also, another improvement is that we applied principal component analysis (PCA) on original feature space before clustering them into clusters. As to the identification of sulci, we semi-manually extracted the sulci fundi based on the methods in Li et al., 2010.

Based on the above-mentioned methods (fiber shape analysis and sulcal fundi extraction), we built $\mathrm{U}$-shape fiber wiring diagrams for different species and verified the finding that the cortical sulci are coursed around by $\mathrm{U}$-shape fibers including those major ones such as precentral sulcus, post-central sulcus, superior temporal sulcus, inferior frontal sulcus, and 
intra-parietal sulcus across species. These U-shape fibers connect their neighboring gyral regions. Also, comparisons across species based on U-shape fibers were conducted and it is found that $\mathrm{U}$-shape fibers are positively correlated with elaboration of gyrification. This study reveals a common pattern of evolutionarily-preserved cortical architecture, and suggests novel insights into the structural and functional organizational principles of the cerebral cortex in primate brains.

\section{Materials and Methods}

\subsection{Data Acquisition and Preprocessing}

Generally, we acquired and used seven diffusion MRI datasets in this work. Dataset 1

(Human DTI Datasets) included eighteen healthy young volunteers who were scanned in a GE 3T Signa MRI system using an 8-channel head coil at the Bioimaging Research Center (BIRC) of the University of Georgia (UGA) under IRB approval were used in this paper. In dataset 2 (Human HARDI Datasets), diffusion-weighted images were acquired from ten adult subjects using a Siemens 3T TIM Trio MR Scanner at UNC Chapel Hill. Diffusion gradients were applied in 120 non-collinear directions with diffusion weighting $b=2000$ $\mathrm{s} / \mathrm{mm}^{2}$. Q-ball model was adopted to deal with fiber crossings. Dataset 3 (Human Connectome Project Datasets) included diffusion MRI data of 68 subjects obtained from the Q1 release of the human connectome project dataset (http:// www.humanconnectomeproject.org/) package. Two fiber tracking methods were applied. 1) Deterministic tracking: single-shell $(b=2000)$ data for each subject was used, onto which qball/HARDI model in Trackvis (http://trackvis.org/) was applied to handle fiber crossings. Angle threshold for fiber tracking was set to be 45 degrees. 2) Probabilistic tracking: the BedpostX in FSL 5 (http://fsl.fmrib.ox.ac.uk/fsl/fslwiki/FDT/UserGuide\#BEDPOSTX) was adopted to deal with the multi-shell data (all the three shells $b=1000,2000$ and 3000 were included). Dataset 4 (Human DSI Datasets) included DSI data of two subjects obtained from the recently released HCP (Human Connectome Project) database (http:// www.humanconnectomeproject.org/). In dataset 5 (Human Fetus DTI Datasets), ex vivo DTI data of a 21 post-conception weeks (pcw) fetus was downloaded from the BrainSpan Atlas of the Developing Human Brain (http://www.brainspan.org). Dataset 6 and dataset 7

(Chimpanzee DTI Datasets \& Macaque DTI Dataset) included MRI scans obtained from fifteen female chimpanzees and twenty female adult macaques, respectively. All chimpanzees and macaques involved were members of a colony at Yerkes National Primate Research Center (YNPRC) in Atlanta, Georgia. All MRI and DTI scans were conducted at the YNPRC of Emory University under IACUC approval. Detailed dataset description and pre-processing can be found in the Supplemental Materials.

\subsection{Joint Analysis of U-Shape Fibers, Sulcal Fundi and Gyral Crest Lines}

Generally, the joint analysis method used in this paper consists of three major steps, as illustrated in Figure 2 with circled numbers. After the preprocessing steps in section 2.1, the fiber tracts (Figure 2(a)) and GM/WM cortical surface (Figure 2(c)) were obtained for each subject. Then, a novel fiber clustering framework conducted in the fiber feature space based on the one in Hu et al., 2010 (detailed in section 2.2.1) was adopted to group fiber tracts into different classes according to their shapes, and further analysis mainly focuses on the U- 
shape classes (Figure 2(b)) in this paper. At the same time, the sulcal fundi (white curves in Figure 2(d)) were semi-manually extracted throughout the GM/WM surface via a similar method as the one in Li, et al., 2010 (detailed in section 2.2.2). In fact, gyral crest lines were also extracted in the similar way (but not shown in Figure 2 in order to make the illustration clear). Finally, U-shape fiber tracts in Figure 2(b) which pass through the nearby neighborhood of sulcal fundi in Figure 1(d) were extracted and labeled with the same color if they course around the same sulcus (Figure 2(e)).

2.2.1 Fiber Clustering - In order to simultaneously obtain U-shape fibers across species and modalities on all datasets, we developed a novel fiber clustering framework based on similar fiber features developed in $\mathrm{Hu}$, et al., 2010 and obtained a feature vector $\vec{V}$ containing 12 elements for each fiber tract. The fiber preprocessing and features extraction are detailed in supplemental materials to make the paper self-contained.

As to the clustering strategy, the method in Hu et al., 2010 lacks the ability to be applied on large-scale datasets, therefore we developed a novel framework to facilitate its application on our cross-species and cross-modality datasets. We took a two-step strategy detailed below to firstly obtain the clustering centers in a small size sample dataset (6000 fibers), and secondly propagate the clustering results across datasets. In order to make sure the cluster results are not biased by any modality (DTI/HARDI/DSI) or species (human/chimpanzee/ macaque), we randomly sampled around 1200 fiber tracts (6000 fiber tracts altogether) from every datasets and use them as samples to be clustered. The sample number within each dataset is even across subjects.

Step (a). Data-driven clustering: the features introduced above were extracted from the 6000 samples. The affinity propagation (AP) clustering algorithm (Frey, et al., 2006) was applied on the similarity matrix of the 6000 sample fibers in the feature space to automatically cluster them into 5 classes. The element of the similarity matrix is defined as the Euclidian distance between two fibers in the features space. The class number is automatically determined by AP algorithm. Altogether, all fibers were clustered into 5 classes (close U, open U, noise, curved line, and straight line). Among the five classes, noisy fibers usually appear to be highly twisted (see Figure 3 for example) and only account for around 2\%, and we therefore discarded those fibers. In Figure 3, we show examples of fibers in the remaining four clusters in the top panel and the percentage bins of all five clusters in the bottom panel. Fibers in clusters \#1 and \#2 are defined as U-shape fibers and those in clusters \#3 and \#4 are defined as Line-shape fibers.

Step (b). Model-driven propagation: We propagate the clustering results obtained on the 6000 samples across datasets. Currently, we adopted the K-means algorithm that uses the five cluster centers obtained in step (a) as the pre-defined centers in the feature space when conducting the K-means algorithm throughout the datasets. The features of all fibers were transformed into the same PCA feature space in step (a) before the K-means algorithm was performed. Again, all fibers classified into the noise cluster were discarded.

\subsubsection{Sulcal Fundi, Gyral Crest Line and Fiber Bundle Extraction-The}

BrainVoyage (www.brainvoyager.com/BrainTutor.html) 3D surface atlas is used as a human 
brain reference by two experts to identify sulci and gyri. As for the macaque and chimpanzee, we referred to the brain atlases in Sincich, et al., 2003 and Bogart, et al., 2012. The open-source ParaView software (http://www.paraview.org/) was used for the experts to interactively extract sulcal fundi and gyral crest lines. First, several sequential vertices (white dots in Figure 4(a)) were selected in the sulcal root region/gyral crest region on the reconstructed surface by referring to the atlas. Then, a similar method with the one in $\mathrm{Li}$ et al., 2010 based on the fast marching algorithm is adopted to connect the sequential vertices into a geodesic curve (the white curve in Figure 4(a)) on the surface. For the convenience of comparison, only major sulci and gyri which are much easier and more reliable to be identified are presented in the paper, which will be detailed in the result section. More details on fast marching based gyral crest lines/sulcal fundi can be found in supplemental materials.

Considering that human brains are approximately three times greater in volume than chimpanzee brains (Rilling, et al., 2007) and five times than macaque brains (MacLeod, et al., 2003), we accordingly extracted the U-shape fiber tracts if they pass through a $3 \mathrm{~mm} / 2 \mathrm{~mm} / 1.8 \mathrm{~mm}$ radius tube formed by the sulcal fundus on human/chimpanzee/macaque brains. As an example, we illustrated this process in Figure 4(b). The reason why we use sulcal fundi as a reference to extract fiber bundles but not the sulcal region is that the reconstructed surface varies in quality from different datasets.

In order to determine which two gyral regions a fiber connects, we compute the minimal distance between $10 \%$ length (10 points) of the termination part of a fiber and all gyral crest lines. If the minimal distance between one termination part and one gyral crest line is less than $5 \mathrm{~mm}$, this termination of the fiber is determined to be connected to this gyral crest line. Here, $5 \mathrm{~mm}$ is used to guarantee that both gyral crest and gyral wall are included in order to avoid the possible dispersion and fanning effects (Heidemann, et al., 2012 and Jbabdi and Johansen-Berg, 2011). If one termination of the fiber locates in the neighborhood of gyrus A while the other locates in the neighborhood of gyrus B, we defined it as connecting gyrus A and B. This method is similar to extracting fiber bundle from the sulcal fundus, but it only takes 10 points at the termination part of a fiber into consideration and determine if those points can pass through $5 \mathrm{~mm}$ radius tube of one gyral crest line.

\section{Results}

In this paper, only those major sulci listed in Supplemental Table 1 were studied. Also, the gyri on the three species used in this paper are listed in Supplemental Table 2. It is worth noting that because we mainly focus on co-localization of U-shape streamline fibers and major sulci in Supplemental Table 1, in order to minimize the impact of fibers connecting hemispheres on both visualization and statistical results, we eliminated those fibers passing through a manually-defined plane (with the visualization from ParaView) which cut the brain into two hemispheres. Those eliminated fibers consist of U-shape fibers passing the corpus callosum and the Line-shape fibers passing through the brain stem. Two subjects in human DSI dataset and ten randomly selected subjects in each of the datasets, human HARDI/human DTI/chimpanzee DTI/macaque DTI datasets, were used for group-wise analysis. One subject in the HCP dataset was used as an example for visual observation. 


\subsection{Method Validation}

In order to compare the performance of the framework with the method in Hu et al., 2010, we randomly selected 400 fiber tracts from all datasets and two experts manually labeled the fiber tracts into U-shape and line-shape. Then, we applied the framework in this paper on the 400 fiber tracts by directly using the propagation step. As to the method in Hu et al., 2010, it is impossible to apply the AP algorithm on all fibers in all datasets and it is also not reasonable to just apply the AP algorithm on those 400 fibers, as this would cause bias due to the lack of samples. Therefore, we included the 400 fibers into other randomly selected ones from all datasets to make the samples as large as $6 \times 10^{3}$. Then the AP algorithm is directly applied onto them. By using those manual labels as pseudo ground truth, we evaluated the clustering performance of both methods. The classification accuracy of the method in this paper is $91 \%$, and the one in Hu et al., 2010 is $93 \%$. This result demonstrates that our framework can preserve high classification accuracy, while having the ability to be applied onto large-scale datasets. As introduced in section 2.2.1, we randomly sampled around 1200 fiber tracts (6000 fiber tracts altogether) from each dataset and used them as samples to be clustered. If we applied the AP algorithm on each dataset separately, the clustering results will be biased by species/dataset variation so that it will be not feasible to compare the results among species/dataset. To better elucidate this point, we randomly selected 6000 fiber tracts from each of the following datasets: human DTI dataset, chimpanzee DTI dataset and macaque DTI dataset. The AP algorithm was applied on each dataset separately and the parameters were tuned accordingly for each dataset to generate five clusters similar to those in Figure 3. The four cluster centers that expect noise cluster for each dataset are shown in Figure 5. In fact, no standard 'close U' shape cluster center as the one in Figure 3 can be found in chimpanzee dataset and macaque dataset (the second and third rows in Figure 5). Therefore, the four cluster centers in chimpanzee dataset (b1-b4) and macaque dataset (c1-c4) were organized according to their similarity to the four cluster centers in human dataset (a1-a4). By comparison, we can observe that 'close U' shape in chimpanzee and macaque datasets are more likely to be identified as 'open U' shape in human dataset, because the 'close U' shape fibers are relatively fewer in non-human brains which will be detailed in 3.4. If we use the clustering results in Figure 5, the comparison among datasets, e.g., the percentage of 'close U' shape fibers in each species, will be biased because fibers classified into 'close $U$ ' cluster in chimpanzee and macaque datasets are more reasonable to classified into 'open U' cluster. Thus, in order to provide a comparable result, we sampled 6000 fiber tracts across all datasets and obtained common cluster centers in Figure 3 based on them.

In addition, we also specified how to choose the parameters in the methods as follows.

1. Cluster number: It is difficult to determine how many types of shapes there would be. So, we adopted the affinity propagation (AP) clustering methods (Frey, et al., 2006), which will automatically determine the cluster number. There is only one parameter $\mathrm{p}$ in the $\mathrm{AP}$ algorithm to control whether the clustering results are in a coarse scale (greater absolute $\mathrm{p}$ value) or a fine scale (smaller absolute $\mathrm{p}$ value). We manually adjusted $\mathrm{p}$ until the algorithm can automatically generate the close $\mathrm{U}$, open $\mathrm{U}$, curved line and straight line clusters (plus one noise cluster and $\mathrm{p}=-13$ ). For example, if we use $\mathrm{p}=-18$, there will be six clusters generated, in which close 
U shape fibers will be split into two clusters, and if $p=-5$, the curved line and straight line shapes will be mixed up.

2. The gyrus/sulcus tube radius: The gyrus tube radius is set to be $5 \mathrm{~mm}$. We manually grouped gyral crest lines into pairs if they are running parallel courses (e.g., the pre-central gyrus \& post-central gyrus pair, the superior frontal gyrus \& middle frontal gyrus pair). Then, the mean distance between the crest lines within each pair is measured and the value is $10.3 \mathrm{~mm}$. Therefore, we used $5 \mathrm{~mm}$ tubes to make less overlapping between gyri so that a fiber tract can be less likely counted as connecting two neighboring gyri at the same time. As for sulcal fundi, we measured the minimal values between any two neighboring sulcal fundi and the value is $6.8 \mathrm{~mm}$. Therefore, we used a $3 \mathrm{~mm}$ tube so that less fiber bundles would be extracted from multiple sulcal fundi. In the future, we plan to enlarge the tube size of sulcal fundi or use a plane beneath sulcus to extract fibers. In this way, we can explore how fiber shapes vary with the increase of the depth.

\subsection{Cross Validation on Human Data}

In this section, we compared U-shape fibers obtained from human brains with different data modalities via different fiber orientation estimation and tracking methods. First, one subject was respectively selected from the DSI, HARDI, DTI and HCP dataset as an example to show the major sulcal fundi. Cross-modality (e.g., DTI, HARDI, DSI and HCP datasets) validation is one of the effective ways to demonstrate the reproducibility of the observation. One advantage of HARDI data, DSI data and HCP data is their ability to resolve crossing fibers at the scale of single MRI voxel. Therefore, the usage of those datasets, though only two subjects were available in DSI dataset currently, is to demonstrate that the phenomenon of U-shape fibers crossing all major sulcal fundi is reproducible and consistent across available data modalities.

The U-shape on the left hemisphere (open U-shape and close U-shape) fibers in DSI are shown in Figure 6, and those for the right hemisphere of DSI and both hemispheres of HARDI, DTI and HCP dataset are shown in Supplemental Figures 2-6

We can observe from Figure 6 and Supplemental Figures 2-6 that U-shape fiber bundle course around the corresponding sulcal fundus in all those figures. Moreover, the fibers' terminations concentrate on the neighborhoods of the gyral crest lines and the distributions of the fibers' terminations also follow the trends of the gyral crest lines. It is worth noting that a variety of factors, like different fiber tracking parameters and different tracking software, can generate different numbers of fibers. For example, fiber bundles in Figure 6 are derived from DSI data via TrackVis software and the total number of fibers is 184,535, while fiber bundle in Supplemental Figures $3 \& 4$ are derived from HARDI data via MEDINRIA software and the total number of fibers is 50,000. This is one of the major factors which make fibers in Figure 6 look much denser. Importantly, the key conclusion that U-shape diffusion imaging derived fibers course around sulci and connect neighboring gyri is consistently true, no matter what datasets and tractography tools/parameters were used. 
We further used the HCP dataset to validate this observation. We selected a group of seed points along the sulcal fundus (the black dots in Figure 7(a)) and used the probabilistic tracking method in FSL on each seed point separately. In Figure 7(a), the left central sulcus was used as an example to show the overlapped probabilistic tracking maps obtained from multiple seed points. In Figure 7(b), only one or two typical seed points were used in order to give a clearer view. It can be seen that voxels with higher probabilistic values form Ushape pathways starting from the seed points and extending the way to neighboring gyral regions. Maps on the right hemisphere can be found in Supplemental Figure 7.

We also measured the U-shape fiber depth using the HCP data which has high resolution $(1.25 \mathrm{~mm} \times 1.25 \mathrm{~mm} \times 1.25 \mathrm{~mm})$ via q-ball model on the single shell $(b=2000)$ of five randomly selected subjects. Then, based on the fiber tracts obtained via deterministic fiber tracking in Trackvis, we computed the minimal distance between each fiber tract and the gray matter/white matter cortical surface. On average, the minimal distance between Ushape fibers and gray matter/white matter boundary is $2.89 \mathrm{~mm}$. This result demonstrates that the fiber bundles are more than 2 voxels away from the boundary and suggest that the fiber dispersion problem may have relatively little impact on U-shape fiber bundles. Also, we adopted the multi-shell model in FSL5 to estimate the fiber orientation of the HCP dMRI data and obtained the orientation dispersion maps. Then, we aligned the U-shape fiber bundles with the dispersion maps and recorded the dispersion values along the pathways. On average, the gray matter dispersion value is 0.0759 which is much greater than white matter (0.0109). The average dispersion value on our $\mathrm{U}$-shape pathways is 0.010 , which is lower than the one in gray matter. This result further demonstrates that $U$-shape fiber bundles discussed in this paper may be less affected by the orientation dispersion problem. Detailed reports of the U-shape depth and dispersion values can be found in Supplemental Tables 3 and 4.

Moreover, our preliminary result on the fetus brain suggests that fiber dispersion may have less statistically effect on fiber termination density. In Figure 8 (a) and 10(b), we compared the fiber termination density map and the maximum principal curvature (MPC) map of a fetus brain. The white arrow highlights a sulcus with more negative MPC value, where less fiber density can be observed. More importantly, the fetus brain is relatively flat rather than highly convoluted, which can be observed by taking a cross-section (the white dashed curve in Figure 8(c)) at the position of red line (frontal lobe) in Figure 8(b). But the fibers beneath the potential sulcus are of U-shape and much deeper than the sulcus (indicated by the yellow dashed arrow). This observation suggests that the deeper parts of those fiber pathways have less possibility to disperse their way to gray matters. Therefore, at the current brain development phase, it might be possible that no strong fiber dispersion effects can be found. Also, based on this observation, we suggest that the development of those U-shape fibers may be independent of the formation of convoluted cortex. Other U-shape fibers in the fetus brain can be found in Supplemental Figure 14.

In order to better illustrate that U-shape fibers connect the neighboring gyri, we computed the group-wise connectivity maps for fiber bundles in the following way. For the fiber bundle extracted from one sulcus, each fiber within it has determined which two gyri it connects. Then, the total number of fibers that connect gyri A and B within this fiber bundle 
is defined as the connectivity strength. Therefore, a connectivity matrix, the row and column of which are the gyrus name and the element of which is the connectivity strength, can be obtained for each sulcus. Then, an average matrix for each sulcus across subjects within each dataset can be directly computed. In order to compactly visualize those connectivity matrices, we firstly picked up the top three strongest connectivities (if there exist more than three connections) from each average matrix, and then integrated those average matrices from different sulci into a connectivity map. In Figure 9, we visualized the U-shape fiber connectivity map of all human datasets. In this figure, gyri are organized on a gyral circle. The connectivities are represented by curves linking gyri inside and outside the gyral circle. Connectivities from the same sulcus are of the same color according to the color bars in Figure 6. The connectivity that links two different gyri is shown inside the gyral circle, and the one that links the same gyrus is outside the circle. The connectivity strength is represented by the width of the curves. To make sure that the maps can be clearly observed, the connectivity strength, the width of the curves were equally amplified within each map. Therefore, direct comparison among the three maps in terms of connectivity strength is improper while it is meaningful within subject.

It is noted that many connectivities within a single gyrus can be found in the human DSI data. For example, the superior frontal gyurs, middle frontal gyrus, and superior parietal lobule are self-connected and fibers course around the nearby sulci, such as the superior frontal sulcus and intra-parietal sulcus. This type of connectivities can also be found in HARDI data, for example the superior frontal gyrus, superior parietal lobule and middle temporal gyrus, and those fibers course around the nearby sulci, such as the superior frontal sulcus, intra-parietal sulcus and superior temporal sulcus. However, this type of connectivity can be barely found in DTI data except a few gyri in frontal lobe such as superior frontal gyrus.

Abundant connectivity can be found within lobes in all three maps such as the frontal lobe (e.g., superior frontal gyrus, middle frontal gyrus and inferior frontal gyrus) and those connecitivties are derived from U-shape fibers coursing around the inferior frontal sulcus, superior frontal sulcus or pre-central sulcus. Gyri in the parietal lobe (e.g., superior parietal gyrus, inferior parietal gyrus and supra-marginal gyrus) are also connected and the connecting fibers course around the intra-parietal sulcus or post-central sulcus.

A few middle-range connectivities can also be found between lobes. In all three modalities, fibers crossing boundary between the parietal lobe and temporal lobe and connecting inferior parietal lobule/supra-marginal gyrus and middle temporal gyrus can be found, and those fibers mostly course around the superior temporal sulcus and a few course around inferior temporal sulcus. In DSI data, fibers crossing boundary between the frontal lobe and parietal lobe and connecting the post-central gyrus/pre-central gyrus and superior parietal lobule/inferior parietal lobule/supra-marginal gyrus can be found, and those fibers mostly course around the central sulcus or post-central sulcus. In DTI data, fibers crossing the boundary between the frontal lobe and parietal lobe can also be found, and they connect the pre-central gyrus and post-central gyrus/superior parietal lobule/inferior parietal lobule and course around the central sulcus and post-central sulcus. Also, a few fibers connecting the parietal lobe (superior parietal lobule) and occipital lobe (superior occipital gyrus) can be 
found, and they course around the inferior temporal sulcus or intra-parietal sulcus.

Additionally, a few long-range U-shape connectivities between the frontal lobe and temporal lobe can be found in all three maps. Those fibers cross multiple sulci like the pre-central sulcus, central sulcus and post-central sulcus, so it is not necessary to determine which sulcus they course around.

Although qualitative comparison among DSI, HARDI and DTI data suggest different levels of diffusion imaging quality, they still share some common connection pattern. For example, those connectivities within frontal lobe and parietal lobe are similar across the modalities.

Further, we quantitatively analyze the average ratio of U-shape fiber number over the total number of fibers extracted from major sulci on all subjects within each datasets. In Table 1, only close U-shape fibers of human brains are considered, i.e., the fibers classified into cluster \#1 in Figure 3. In Table 2, U-shape fibers of human brains classified into cluster \#2 are also included to obtain the U-shape-to-all ratio. The ratio was computed on each subject individually, and then the average ratio within each dataset is reported in tables in this section.

It can be seen from Table 1 that very few (left hemisphere: 0.03 , right hemisphere: 0.02 ) close U-shape fibers coursing around the central sulcus can be found in DSI data, and the ratio values in the other two modalities are also low (HARDI: left: 0.13, right: 0.16; DTI: left: 0.17 , right: 0.14 ) in the central sulcus compared to other sulci. Dense close U-shape fibers can be found in the intra-parietal sulcus in both DSI (left: 0.29, right: 0.31) and HARDI (left: 0.20, right: 0.17) data, while they are not dense in DTI (left: 0.13, right: 0.14) data. Dense fibers can be found in the pre-central sulcus and superior temporal sulcus in all three modalities (shaded by gray bars). After adding open U-shape fibers (Table 2), the Ushape fibers take up around $40 \%$ of all fibers. The differences of ratio from major sulci are decreased across modalities compared to Table 1, indicating the overall distribution of Ushape fibers across the brain. In comparison, less U fibers can be found in the central sulcus, superior temporal sulcus and transverse occipital sulcus (shaded by gray bars).

\subsection{Joint Analysis of Primate Data}

In this section, we randomly selected one subject from the chimpanzee DTI dataset to show the major sulcal fundi and the U-shape (open U-shape and close U-shape) fibers on the left hemisphere in Figure 10 (the right hemisphere of chimpanzee DTI and both hemispheres of macaque are shown in Supplemental Figures 8-10). In Figure 11, we visualized the groupwise U-shape fiber connectivity map of the two species. In general, U-shape fiber bundle can also be found to be coursing around the correspondent sulcal fundus. The fibers extend their way to the neighborhood of the gyral regions.

In Figure 11(a), a few short range U-shape connectivities can be found within a single gyrus (superior frontal gyrus, post central gyrus and superior parietal lobe). Those fibers course around superior frontal sulcus/pre-central sulcus, central sulcus/post central sulcus and intraparietal sulcus. U-shape connectivities can also be found within the frontal lobe (superior frontal gyrus, middle frontal gyrus, inferior frontal gyrus and pre-central gyrus) and parietal lobule (superior parietal lobule and supra-marginal gyrus). Those fibers course around the 
superior frontal/inferior frontal/pre-central sulcus and intra-parietal sulcus respectively. Inter-lobe middle-range $\mathrm{U}$ shape connectivities can also be found between the frontal lobe and parietal lobe (between pre-central gyrus and supra-marginal gyrus, and between superior frontal gyrus and post-central gyrus) and those fibers mainly course around central sulcus. Virtually weak long-range connectivity, like fibers connecting the frontal lobe and temporal lobe in human brains, can be found in chimpanzee brains.

In the macaque DTI data (Figure 11(b)), intra-gyrus U-shape connectivity exists within precentral gyrus and superior temporal gyrus, and course around arcuate sulcus and superior temporal sulcus respectively. Intra-lobe connectivity exists within the frontal lobe (superior frontal gyrus, middle frontal gyrus, inferior frontal gyrus and pre-central gyrus) and parietal lobe (superior parietal lobule, supra-marginal gyrus and angular gyrus). Those fibers course around the arcuate/principal sulcus and intra-parietal sulcus, respectively. Inter-lobe connections can be found between the parietal lobe and temporal lobe, that is, fibers course around the intra-parietal sulcus and connect superior parietal lobule and superior temporal gyrus/angular gyrus. Particularly, the connectivity between the pre-central gyrus and postcentral gyrus coursing around central sulcus can be found and relatively stronger than others. No long-range U-shape connectivity can be found. This result is consistent with the one in Thiebaut de Schotten, et al., 2011.

In summary, the figures and results in sections 3.2 and 3.3 and in Supplemental Figures 2-10 consistently demonstrated the U-shape fibers coursing around major sulci across the whole cortex, across data modalities, and across three primate species. These results reveal that the common pattern of $\mathrm{U}$-shape fibers coursing around sulci is evolutionarily-preserved in cortical architectures.

Similarly, the close U-shape fiber ratios and U-shape fiber ratios (including open $\mathrm{U}$ ) of chimpanzees and macaques are shown in Table 3 and Table 4. Generally, denser close Ushape fibers (see Ratio 1) can be found in the inferior post-central sulcus (left: 0.11, right: 0.24 ) and inferior pre-central sulcus (left: 0.11, right: 0.32) in chimpanzees, while fewer can be found in the lunate sulcus (left: 0.02, right: 0.08). Again, when adding open U-shape fibers into consideration (see Ratio 2 ), we can find that $U$-shape fibers are relatively evenly distributed across sulci and take up around $40 \%$ of all fibers. As to macaque data, denser close U-shape fibers course around the intraparietal sulcus (left: 0.18, right: 0.17 ) and superior temporal sulcus (left: 0.15 , right: 0.14 ), while fewer can be found in the arcuate sulcus (left: 0.08 , right: 0.05 ). Dense U-shape fibers (including open U shape) can be found coursing around the arcuate sulcus (left: 0.43 , right: 0.40 ) and principal sulcus (left: 0.43 , right: 0.41), while fewer can be found in the central sulcus (left: 0.33, right: 0.33).

\subsection{Comparison across Species}

In order to quantitatively compare the fiber shapes across datasets, we computed the ratio of fibers classified into each of the four clusters in Figure 3 over the total number of fibers within each subject, and show the results in Supplemental Figure 11 (DSI and HARDI data were used as reference). Here, fibers connecting two hemispheres are not considered. Generally, human brains (HARDI and DTI datasets) have greater close U-shape ratios and open $\mathrm{U}$ ratios than chimpanzees and macaques. Although human DSI data has fewer close 
U-shape fiber, it prevails over others in open U-shape. Totally, U-shape ratios (including close U-shape and open U-shape) in human brains are 0.45 (DSI), 0.43 (HARDI) and 0.35 (DTI), which are greater compared to chimpanzees $(0.23)$ and macaques $(0.24)$. The ratios of line shape (including curved line and straight line) fibers in chimpanzee (0.77) and macaque (0.76) brains surpass those in human brains (DSI: 0.55, HARDI: 0.57 and DTI: 0.65). Statistical t-test was conducted on U-shape fibers ratios (including close U-shape and open U-shape) between species. Considering the data modality impact, t-test is only conducted on the DTI data of the three species. The p-values are 0.008 and 0.035, respectively, for human-chimpanzee and human macaque pairs when two-tailed test is performed, and the p-values are 0.011 and 0.021 in right-tailed tests (human ratio is greater than chimpanzee/macaque ratio). This result indicates that fibers in human brains are more deflected from the straight courses than chimpanzee and macaque brains.

\section{Discussion and Conclusion}

In this work, three modalities of diffusion imaging data (DSI, HARDI and DTI) and deterministic fiber tracking methods (TrackVis and MedINRIA) were used to reconstruct the fiber pathways. The former two imaging techniques (DSI and HARDI) were developed in order to better resolve crossing fibers at the scale of single MRI voxels (Tuch et al., 2002; Wedeen et al., 2005; Wedeen et al., 2012, Ozarslan et al., 2003). Many previous studies have validated the effectiveness of these imaging techniques via either phantom study (Poupon et al., 2008) or autoradiography-based comparison study (Schmahmann et al., 2007). As an example, we further examined the orientation distribution functions (ODFs) derived from human DSI data in Supplemental Figure 12 to visually verify the effectiveness of deterministic tracking methods, as well as the fact that reconstructed U-shape fiber coursing around the sulcal fundi. Specifically, we extracted voxels around two sulcal fundi (superior temporal sulcus and intra-parietal sulcus), and jointly showed the fundi and ODFs derived from those nearby voxels in Supplemental Figure 12. Two different views were taken for each sulcus. It can be clearly appreciated that orientations of the local maxima on ODF profiles are roughly tangential to the sulcal fundus and form a sweeping flow around the fundus. This result visualizes our previous finding that $\mathrm{U}$-shape fibers course around sulcal fundi.

We are also aware of the possible biases of streamline fiber tracking methods and low-level modeling approaches that might cause false positives/negatives when they approach the gray matter cortex. Dispersion and fanning of white matter fibers can be observed near the gray/ white matter boundary (Heidemann, et al., 2012 and Jbabdi and Johansen-Berg, 2011).

Meanwhile, in Sotiropoulos, et al., 2013, comparison has been made between diffusion MRI and histology images on macaque cortex. It is inspiring that DTI-derived fiber orientations are consistent with histology data within the white matter area, which supports the validity of DTI and the results in this paper. Based on these studies, although diffusion MRI lacks the ability of detecting dispersion and fanning of fiber terminations entering gray matter in gyral walls, the overall shape of fiber tracts may not be impact severely and we suggest that the existence of observed U-shape fibers across whole brain are not likely to be the result of the bias of tracing approach. Moreover, Sotiropoulos and his colleagues also demonstrate that both the modalities predict reduced dispersion near the fundus of a sulcus. Therefore, 
when we observe the sulcal fundus in this paper it appears more confident to suggest the conclusion that those fiber bundles course around the sulcal fundus. To better illustrate this point, we selected four sulci and jointly visualize the white matter surface and ODFs reconstructed from DSI data in Supplemental Figure 13. Cross sections (white curves) were used to better illustrate the location of sulcal roots and gyral walls. Black arrows highlight some ODFs beneath the sulcal roots while yellow arrows highlight some ones near the gyral walls. We can observe that those ODFs beneath the sulcal roots are of the polarized shape, suggesting less dispersion of fibers coursing around the sulcal root, while anisotropic ODFs can be found near the gyral walls, suggesting more dispersion and fanning when they entering the grey matter. Also, the results in section 3.2 including the minimal distance between U-shape fibers and gray matter/white matter boundary is $2.89 \mathrm{~mm}$, more than 2 voxels away from the boundary, the average dispersion value on our U-shape pathways is 0.010 , lower than the one in gray matter (0.0759) and our preliminary result on the fetus brain (Figure 8), suggest that the fiber dispersion problem may have relatively little impact on U-shape fiber bundles.

Although we suggested some conclusions based on the current datasets and up-to-date fiber tracking and analysis methods in this paper, further studies including higher resolution diffusion MRI data and other modalities like histology data are needed in the future to carefully further and complete the interpretation of co-localization of fibers and sulci.

From the methodological point of view, most of the current fiber clustering methods (e.g., O'Donnell et al., 2007; O'Donnell et al., 2013; Wang et al., 2011; Wassermann et al., 2010; Visser et al., 2011) were developed to segment streamlines into anatomically relevant bundles so that the fiber clusters may have anatomical plausibility. However, the goal in this paper is to characterize U-shape fiber, and thus we are interested in how to describe the shape of fibers so that U-shape fibers across the whole brain can be identified. Therefore, it is acceptable in this paper that $\mathrm{U}$ shape fibers in the frontal lobe and the occipital lobe would be clustered into the same class. This fiber shape based clustering cannot be achieved by other methods as far as we know, which can only group adjacent fibers into clusters and depict fiber bundle boundaries but cannot generate specific $U$ shape fiber clusters as we need. Also, current fiber analysis methods summarized in O'Donnell et al., 2013, including the one developed by ourselves in Hu et al., 2010, mainly focused on clustering fiber tracts into bundles. They relied on constructing a similarity matrix, the element of which is the similarity measure between any two fiber tracts. The computation load is limited by the size of the similarity matrix. However, our goal is comparing the fiber shapes across species and across datasets (referred by us as 'large scale') and there will be millions of fiber tracts to deal with. An alternative approach could be clustering fiber tracts of subjects individual by individual and then collecting the individual clustering results and making the comparison. However, the problem is that individual clustering results could be biased by individual variation. The similar problem can also be met if we apply clustering methods onto each species separately. In this paper, instead of directly applying clustering methods onto all fibers, we sampled a subset of fibers across datasets and species and used the affinity propagation clustering methods to determine the common cluster centers, which are the five fiber shapes. The five shapes were derived across datasets and species, and therefore they can be used as common templates and all fiber tracts from different species can be compared 
by measuring the similarity to those common templates. Based on this concept, we used the $\mathrm{k}$-means method to propagate the five-fiber-shape labels across all datasets. Therefore, this scheme effectively deals with the 'large-scale' dataset problem, and makes the clustering results comparable, as well as without introducing the 'cross-species' bias.

When comparison was conducted among the three diffusion imaging modalities (DSI, HARDI and DTI) in human brains, more self-connected gyrus, that is, the gyrus connected by two terminations of the same fibers, can be found in DSI and HARDI data other than in DTI data. Also we observed that middle-range connectivity of DTI data is stronger than short-range connectivity within the same subject, while short-range connectivity is stronger than middle-range connectivity within DSI data and HARDI data. This observation may indicate that the capacity of DTI to detect short-range and highly curved fibers is relatively low. The reason might be that the co-localization of highly curved short-range fibers (close U-shape suggested in this paper) and middle-range fibers might cause many crossings within voxels, and the ratio of highly curved short-range fibers is lower than middle-rang ones. Therefore, the inability of DTI to detect crossing fibers may bring more false negatives (ineffective tracking of pathways that do exist) in tractography reconstruction of this class of fiber. However, the existence of U-shape fibers which course around sulcus and connect neighboring gyri is consistently evident and has been cross-validated among multiple diffusion imaging modalities and cross-species datasets.

The phenomenon that U-shape streamline fiber courses along sulcal fundi and connects neighboring gyri can be observed apparently near sulci like the intra-parietal sulcus where $\mathrm{U}$ shape fibers dominate. Even in sulci like the central sulcus where U-shape fibers take a small portion, line-shape fibers still course around its fundus in a similar way, as illustrated in Figure 1(a). The underlying reason of this phenomenon may be due to the inability of the three diffusion imaging techniques, which should be investigated in the future. Moreover, the co-localization of U-shape fibers and gyri may also suggest a specific role that U-shape fiber plays in gyrification procedure. In Hu et al., 2010, the preliminary results showed the correlation between cortical folding pattern and fiber shapes. This figure statistically showed that the close U-shape fibers are more like to terminate in the neighborhood of peak/ridgeshape gyri regions. This result was reproduced in DSI data as shown in Supplemental Figure 15. Though fibers may disperse and fan when they entering the gray matter of gyral wall, we can still observe that gyri regions around the $\mathrm{U}$-shape fiber terminations are more highly convoluted than other regions. This observation is also mentioned in Wedeen et al. 2012, in which the authors found that path orientation was aligned with gyral topography in the arcuate sulcus and they suggested that the relation of fiber structure and cortical folding merits further investigation.

The comparison result among species that U-shape fibers take over a larger portion in human brain than chimpanzee and macaque brains can be better explained, given our previous hypothesis that $\mathrm{U}$-shape fiber might have a positive correlation with the elaboration of gyrification. Such observation can also be found in our previous study (Chen, et al., 2012), in which more elaborate folding pattern and fiber bundle orientation pattern have been validated along the phylogeny scale, and in Wedeen et al., 2012 where the authors suggested that cross-species homology was strong and showed emergence of complex gyral 
connectivity by continuous elaboration of this grid structure. From the functional perspective of view, many long-range comparison studies have reported some major differences between human and other primates. For example, in Thiebaut de Schotten, et al., 2012 and James, et al., 2008, major differences were found for the arcuate fasciculus, which may underlie unique human language functions. This result can also be observed in our connectivity map in Figure 9(c), Figure 11(a) and Figure 11(b). Nevertheless, much more joint comparison work is warranted in the future to explain the result in this paper that $\mathrm{U}$ shape fibers are less in overall regions in non-human brain, from both structural and functional perspectives.

Finally, we would like to point out that this study and its results should be interpreted with caution that diffusion imaging including DSI, HARDI and DTI is limited to connectivity mapping at the macro-scale. In particular, it is noteworthy to point out that current diffusion imaging techniques (DTI, HARDI and DSI) have limitations in spatial resolution, dealing with crossing fibers, and mapping axonal fibers around the boundaries between gray matter and white matter. Essentially, current diffusion imaging techniques are far from being able to map the complete complex fiber pathways and connectional architectures of the cerebral cortex (Schmahmann and Pandya, 2006). Therefore, in the future, micro-scale bioimaging techniques, e.g., the recently developed series two-photon tomography imaging (Ragan et al., 2012), should be used to further examine the identified U-shape streamline fiber connection patterns among cortical gyri and sulci via macro-scale neuroimaging techniques. In general, integration of structural connectivity imaging techniques at multiple scales is a very promising future research direction.

\section{Supplementary Material}

Refer to Web version on PubMed Central for supplementary material.

\section{Acknowledgments}

T Liu was supported by the NIH Career Award EB 006878, NIH R01 DA033393, NSF CAREER Award IIS-1149260, and The University of Georgia start-up research funding. T Zhang was supported by 'Scholarship Award for Excellent Doctoral Student granted by Ministry of Education of China' and 'Excellent Doctorate Foundation of Northwestern Polytechnical University'. L Li and X Hu were supported by NIH PO1 AG026423 and NIH R01 DA033393. D Zhang was supported by China Government Scholarship. L Guo was supported by NSFC-61273362. The authors would like to thank the Human Connectome Project (http:// www.humanconnectomeproject.org/) for sharing the DSI dataset. The authors would like to thank the anonymous reviewers for their constructive comments.

\section{References}

Andersson JL, Skare S, Ashburner J. How to correct susceptibility distortions in spin-echo echo-planar images: Application to diffusion tensor imaging. Neuroimage. 2003; 20:870-888. [PubMed: 14568458]

Behrens TE, Johansen-Berg H, Woolrich MW, Smith SM, Wheeler-Kingshott CA, Boulby PA, Barker GJ, Sillery EL, Sheehan K, Ciccarelli O, Thompson AJ, Brady JM, Matthews PM. Non-invasive mapping of connections between human thalamus and cortex using diffusion imaging. Nat Neurosci. 2003; 6(7):750-7. [PubMed: 12808459]

Basser PJ, Pierpaoli C. Microstructural and physiological features of tissues elucidated by quantitativediffusion-tensor MRI. Journal of Magnetic Resonance Series B. 1996; 111(3):209-219. [PubMed: 8661285] 
Berghuis P, Rajnicek AM, Morozov YM, Ross RA, Mulder J, Urban GM, Monory K, Marsicano G, Matteoli M, Canty A. Hardwiring the brain: endocannabinoids shape neuronal connectivity. Science. 2007; 316:1212-1216. [PubMed: 17525344]

Bogart SL, Mangin JF, Schapiro SJ, Reamer L, Bennett AJ, Pierre PJ, Hopkins WD. Cortical sulci asymmetries in chimpanzees and macaques: a new look at an old idea. Neuroimage. 2012; 61(3): 533-41. [PubMed: 22504765]

Brown, M.; Keynes, R.; Lumsden, A. The developing brain. Oxford: Oxford University Press; 2002.

Catani M, Jones DK, Donato R, Ffytche DH. Occipito-temporal connections in the human brain. Brain. 2003; 126:2093-2107. [PubMed: 12821517]

Chen H, Zhang T, Guo L, Li K, Yu X, Li L, Hu X, Han J, Hu X, Liu T. Coevolution of gyral folding and structural connection patterns in primate brains. Cerebral Cortex. 2012 Epub ahead of print.

Mori S. Principles of diffusion tensor imaging and its applications to basic neuroscience research. Neuron. 2006; 51(5):527-539. [PubMed: 16950152]

Chédotal A, Richards LJ. Wiring the brain: the biology of neuronal guidance. Cold Spring Harb Perspect Biol. 2010; 2(6):a001917. [PubMed: 20463002]

Conturo TE, Lori NF, Cull TS, Akbudak E, Snyder AZ, Shimony JS, McKinstry RC, Burton H, Raichle ME. Tracking neuronal fiber pathways in the living human brain. Proc Natl Acad Sci U S A. 1999; 96:10422-10427. [PubMed: 10468624]

Corouge I, Gouttard S, Gerig G. Towards a shape model of white matter fiber bundles using diffusion tensor MRI. IEEE Int Symp Biomed Imag. 2004:344-347.

Dennis DM, O'Leary DD, Chou S, Sahara S. Area patterning of the mammalian cortex. Neuron. 2007; 56(2):252-269. [PubMed: 17964244]

Frey BJ, Dueck D. Clustering by passing messages between data points. Science. 2006; 315:972-976. [PubMed: 17218491]

Fu AMN, Yan H, Huang K. A curve bend function based method to characterize contour shapes. Pattern Recognition. 1997; 30(10):1661-1671.

Gerig G, Gouttard S, Corouge I. Analysis of brain white matter via fiber tract modeling. Conf Proc IEEE Eng Med Biol Soc. 2004; 6:4421-4. [PubMed: 17271286]

Heidemann RM, Anwander A, Feiweier T, Knösche TR, Turner R. k-space and q-space: combining ultra-high spatial and angular resolution in diffusion imaging using ZOOPPA at 7 T. Neuroimage. 2012; 60(2):967-78. [PubMed: 22245337]

Hu, X.; Guo, L.; Zhang, T.; Li, G.; Nie, J.; Jiang, X.; Zhang, D.; Liu, T. Joint analysis of fiber shape and cortical folding patterns. ISBI'10 (Proceedings of the 2010 IEEE international conference on Biomedical imaging: from nano to macro; 2010. p. 1165-1168.

Jbabdi S, Johansen-Berg H. Tractography: where do we go from here? Brain Connect. 2011; 1(3):16983. 2011. [PubMed: 22433046]

Kimmel R, Sethian JA. Computing geodesic paths on manifolds. Proc Natl Acad Sci. 1998; 95(15): 8431-8435. [PubMed: 9671694]

LeBihan D. Looking into the functional architecture of the brain with diffusion MRI. Nature Rev Neurosci. 2003; 4:469-480. [PubMed: 12778119]

Li G, Guo L, Nie J, Liu T. An automated pipeline for cortical sulcal fundi extraction. Med Image Anal. 2010; 14(3):343-59. [PubMed: 20219410]

Li L, Rilling JK, Preuss TM, Glasser MF, Hu X. The effects of connection reconstruction method on the interregional connectivity of brain networks via diffusion tractography. Hum Brain Mapp. 2012; 33(8):1894-913. [PubMed: 21928316]

Liu T, Li H, Wong K, Tarokh A, Guo L, Wong S. Brain tissue segmentation based on DTI data. NeuroImage. 2007; 38(1):114-23. [PubMed: 17804258]

Liu T, Nie J, Tarokh A, Guo L, Wong S. Reconstruction of central cortical surface from MRI brain images: Method and Application. NeuroImage. 2008; 40(3):991-1002. [PubMed: 18289879]

MacLeod CE, Zilles K, Schleicher A, Rilling JK, Gibson KR. Expansion of the neocerebellum. Hominoidea J Hum Evol. 2003; 44(4):401-29.

Nie J, Guo L, Li K, Wang Y, Chen G, Li L, Chen H, Deng F, Jiang X, Zhang T, Huang L, Faraco C, Zhang D, Guo C, Yap PT, Hu X, Li G, Lv J, Yuan Y, Zhu D, Han J, Sabatinelli D, Zhao Q, Miller 
LS, Xu B, Shen P, Platt S, Shen D, Hu X, Liu T. Axonal fiber terminations concentrate on gyri. Cerebral Cortex. 2011 Epub ahead of print.

O'Donnell LJ, Kubicki M, Shenton ME, Dreusicke MH, Grimson WE, Westin CF. A method for clustering white matter fiber tracts. AJNR Am J Neuroradiol. 2006; 27(5):1032-6. [PubMed: 16687538]

O'Donnell L, Westin C. Automatic tractography segmentation using a highdimensional white matter atlas. IEEE Trans Med Imaging. 2007; 26(11):1562-1575. [PubMed: 18041271]

O'Donnell LJ, Golby AJ, Westin CF. Fiber clustering versus the parcellation-based connectome. Neuroimage. 2013; 80:283-9. [PubMed: 23631987]

O'Leary DD. Do cortical areas emerge from a protocortex? Trends Neurosci. 1989; 12:400-406. [PubMed: 2479138]

Ozarslan E, Mareci TH. Generalized diffusion tensor imaging and analytical relationships between diffusion tensor imaging and high angular resolution diffusion imaging. Magn Reson Med. 2003; 50(5):955-65. [PubMed: 14587006]

Passingham RE, Stephan KE, Kötter R. The anatomical basis of functional localization in the cortex. Nature Review Neuroscience. 2002; 3(8):606-16.

Fillard P, Arsigny V, Pennec X, Ayache N. Clinical DT-MRI Estimation, Smoothing and Fiber Tracking with Log-Euclidean Metrics. IEEE Transactions on Medical Imaging. 2007; 26(11): 1472-1482. [PubMed: 18041263]

Poupon C, Rieul B, Kezele I, Perrin M, Poupon F, Mangin JF. New diffusion phantoms dedicated to the study and validation of high-angular-resolution diffusion imaging (HARDI) models. Magn Reson Med. 2008; 60(6):1276-83. [PubMed: 19030160]

Rakic P. Specification of cerebral cortical areas. Science. 1988; 241:170-176. [PubMed: 3291116]

Ragan T, Kadiri LR, Venkataraju KU, Bahlmann K, Sutin J, Taranda J, Arganda-Carreras I, Kim Y, Seung HS, Osten P. Serial two-photon tomography for automated ex vivo mouse brain imaging. Nat Methods. 2012; 9(3):255-8.10.1038/nmeth.1854 [PubMed: 22245809]

Rash BG, Grove EA. Area and layer patterning in the developing cerebral cortex. Curr Opin Neurobiol. 2006; 16:25-34. [PubMed: 16426837]

Richman D, Stewart R, Hutchinson J, Caviness V Jr. Mechanical model of brain convolutional development. Science. 1975; 189:18-21. [PubMed: 1135626]

Rilling JK, Glasser MF, Preuss TM, Ma X, Zhao T, Hu X, Behrens TE. The evolution of the arcuate fasciculus revealed with comparative DTI. Nat Neurosci. 2008; 11(4):426-8. [PubMed: 18344993]

Rilling JK, Barks SK, Parr LA, Preuss TM, Faber TL, Pagnoni G, Bremner JD, Votaw JR. A comparison of resting-state brain activity in humans and chimpanzees. Proceedings of the National Academy of Sciences. 2007; 104(43):17146-17151.

Schmahmann JD, Pandya DN, Wang R, Dai G, D'Arceuil HE, de Crespigny AJ, Wedeen VJ. Association fibre pathways of the brain: parallel observations from diffusion spectrum imaging and autoradiography. Brain. 2007; 130(3):630-53. [PubMed: 17293361]

Schmahmann, J.; Pandya, D. Fiber Pathways of the Brain. Oxford Press; 2006.

Sethian JA. A fast marching level set method for monotonically advancing fronts. Proc Natl Acad Sci USA. 1996; 93:1591-1595. [PubMed: 11607632]

Sincich LC, Adams DL, Horton JC. Complete flatmounting of the macaque cerebral cortex. Vis Neurosci. 2003; 20(6):663-86. [PubMed: 15088719]

Smith SM. Fast robust automated brain extraction. Hum Brain Mapp. 2002; 17:143-155. [PubMed: 12391568]

Smith SM, Brady JM. SUSAN - A new approach to low level image processing. Int J Comput Vision. 1997; 23:45-78.

Sotiropoulos SN, Chen C, Dikranian K, Jbabdi S, Behrens TEJ, Van Essen DC, Glasser MF. Comparison of diffusion MRI predictions and histology in the macaque brain. Proc Intl Soc Mag Reson Med. 2013; 21:835.

Sur M, Rubenstein JL. Patterning and plasticity of the cerebral cortex. Science. 2005; 310:805-810. [PubMed: 16272112] 
Tao X, Prince JL, Davatzikos C. Using a statistical shape model to extract sulcal curves on the outer cortex of the human brain. IEEE Trans Med Imaging. 2002; 21(5):513-24. [PubMed: 12071622]

Thiebaut de Schotten M, Dell'Acqua F, Valabregue R, Catani M. Macaque to human comparative anatomy of the frontal lobe association tracts. Cortex. 2012; 48(1):82-96. [PubMed: 22088488]

Tuch DS, Reese TG, Wiegell MR, Makris N, Belliveau JW, Wedeen VJ. High angular resolution diffusion imaging reveals intravoxel white matter fiber heterogeneity. Magn Res Med. 2002; 48(4):577-582.

Visser E, Nijhuis E, Buitelaar J, Zwiers M. Partition-based mass clustering of tractography streamlines. NeuroImage. 2011; 54(1):303-312. [PubMed: 20673849]

Wang X, Grimson W, Westin C. Tractography segmentation using a hierarchical Dirichlet processes mixture model. NeuroImage. 2011; 54(1):290-302. [PubMed: 20678578]

Wassermann D, Bloy L, Kanterakis E, Verma R, Deriche R. Unsupervised white matterfiber clustering and tract probability map generation: applications of a Gaussian process framework for white matterfibers. NeuroImage. 2010; 51(1):228. [PubMed: 20079439]

Wedeen VJ, Hagmann P, Tseng WY, Reese TG, Weisskoff RM. Mapping complex tissue architecture with diffusion spectrum magnetic resonance imaging. Magn Reson Med. 2005; 54:1377-86. [PubMed: 16247738]

Wedeen VJ, Rosene DL, Wang R, Dai G, Mortazavi F, Hagmann P, Kaas JH, Tseng WY. The geometric structure of the brain fiber pathways. Science. 2012; 335(6076):1628-34. [PubMed: 22461612]

Welker W. Why does cerebral cortex fissure and fold? A review of determinants of gyri and sulci. Cereb Cortex. 1990; 8:3-136.

Zhang D, Guo L, Zhu D, Li K, Li L, Chen H, Zhao Q, Hu X, Liu T. Diffusion Tensor Imaging Reveals Evolution of Primate Brain Architectures. Brain Structure and Function. 2012 in press.

Zhang Y, Brady M, Smith S. Segmentation of brain MR images through a hidden Markov random field model and the expectation-maximization algorithm. IEEE Trans Med Imaging. 2001; 20:4557. [PubMed: 11293691]

Zhu D, Li K, Faraco C, Deng F, Zhang D, Jiang X, Chen H, Guo L, Miller LS, Liu T. Optimization of functional brain ROIs via maximization of consistency of structural connectivity profiles. NeuroImage. 2011; 59(2):1382-1393. [PubMed: 21875672]

Zhu D, Li K, Faraco C, Deng F, Zhang D, Jiang X, Chen H, Guo L, Miller LS, Liu T. Discovering Dense and Consistent Landmarks in the Brain. Neuroimage. 2012; 59(2):1382-93. [PubMed: 21875672] 


\section{Research Highlights}

1. A novel computational framework for characterization of U-shape fibers.

2. Identification and characterization of U-shape fibers from DTI/HARDI/DSI data.

3. Characterization of U-shape fibers from human, chimpanzee and macaque brains.

4. Most U-shaped fibers connect neighboring gyri by coursing along cortical sulci.

5. Reveal an evolutionarily-preserved common pattern of $U$-shape fiber connectivity. 


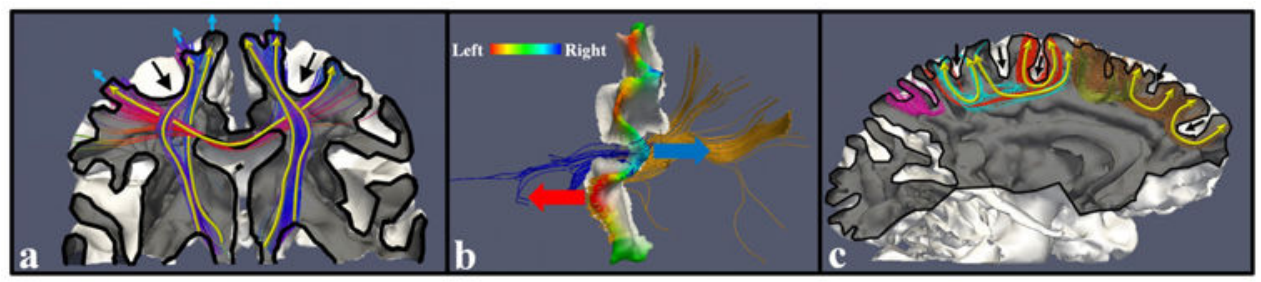

Figure 1.

(a) Joint representation of cortical gyral folding (derived from MRI) and streamline fiber connection patterns (derived from DSI) on a coronal section through the middle part of human brain. Sulcal regions are highlighted by black arrows. The direction of blue arrows suggests the normal orientation of the gyri. (b) Demonstration that convex cortical gyral shapes are connected by dense fibers orienting along the tangent folding direction (indicated by arrows) to the cortical sphere of the gyral crests. The surface patch is color-coded by the fiber termination orientation according to the color bar. (c) Joint representation of cortical gyral folding (derived from MRI) and U-shaped streamline fiber connection patterns (derived from DSI) on a sagittal section through the superior level of central sulcus. Yellow curves highlight the trajectories of the dominant fiber bundles and the yellow arrows indicate the orientation of the fibers' terminations which penetrate the cortical surface. 


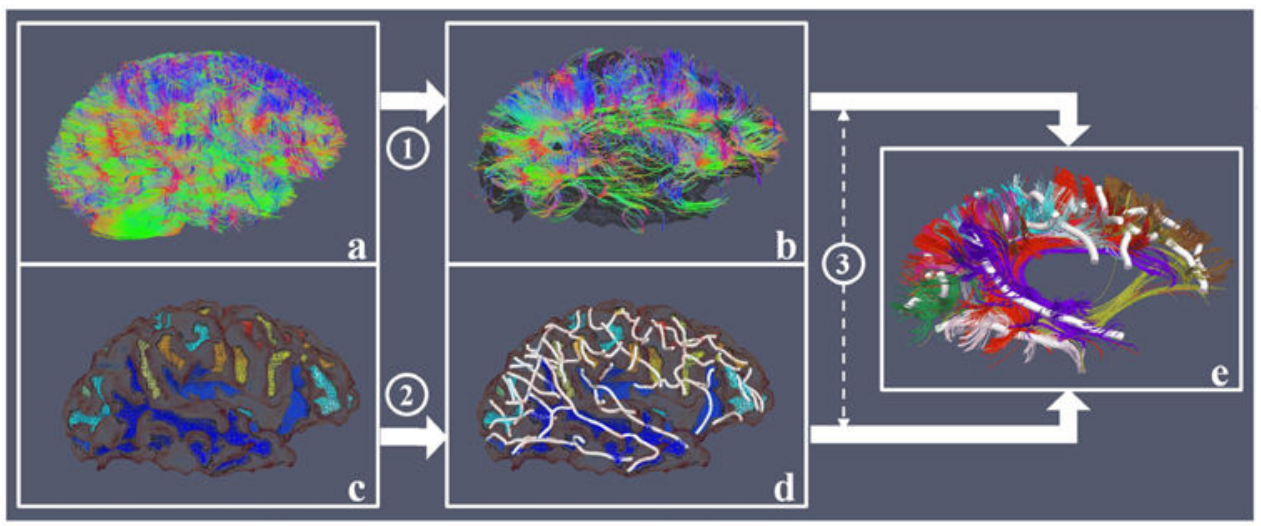

Figure 2.

The flowchart of the fiber shape analysis methods. (a) Whole-brain white matter fiber tractography; (b) U-shape fibers; (c) Reconstructed GM/WM surface with gyral region color-coded by brown and sulcal region color-coded by other colors; (d) Sulcal fundi (white curves); (e) U-shape fiber bundles extracted from sulcal fundi. Step 1: fiber clustering method detailed in section 2.2.1; Step 2: Sulcal fundi extraction method detailed in section 2.2.2; Step 3: Fiber bundles extraction around sulcal fundi detailed in section 2.2.2. 


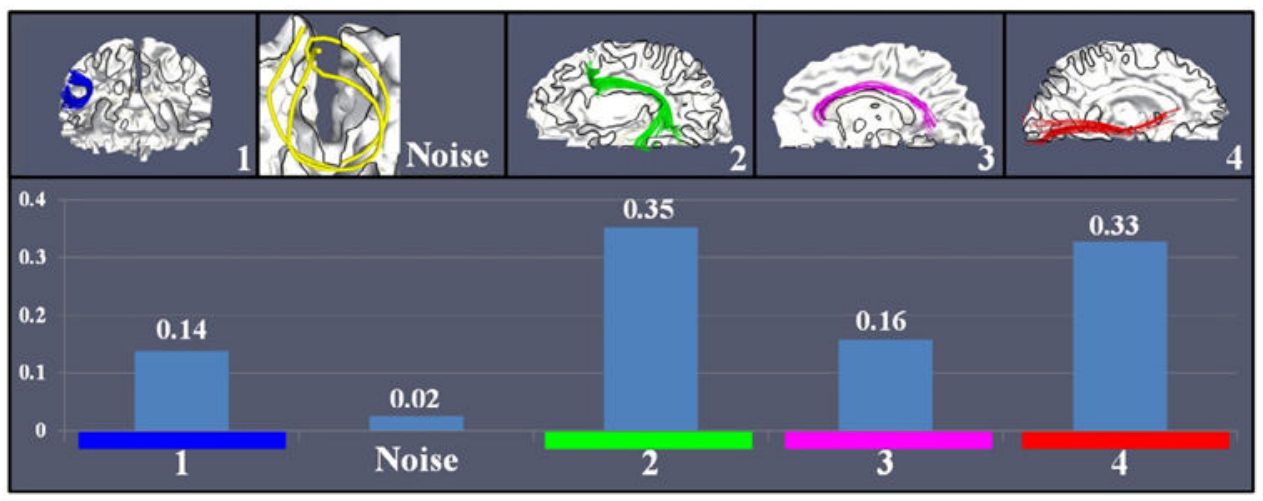

Figure 3.

The bottom panel shows the ratio of fiber number in each class over the total number (in the sample dataset). Example fibers in the five classes analyzed in this paper are shown in the top panel, and fibers are color-coded according to the corresponding color bars in the bottom panel. The noisy fiber is shown in yellow color. 


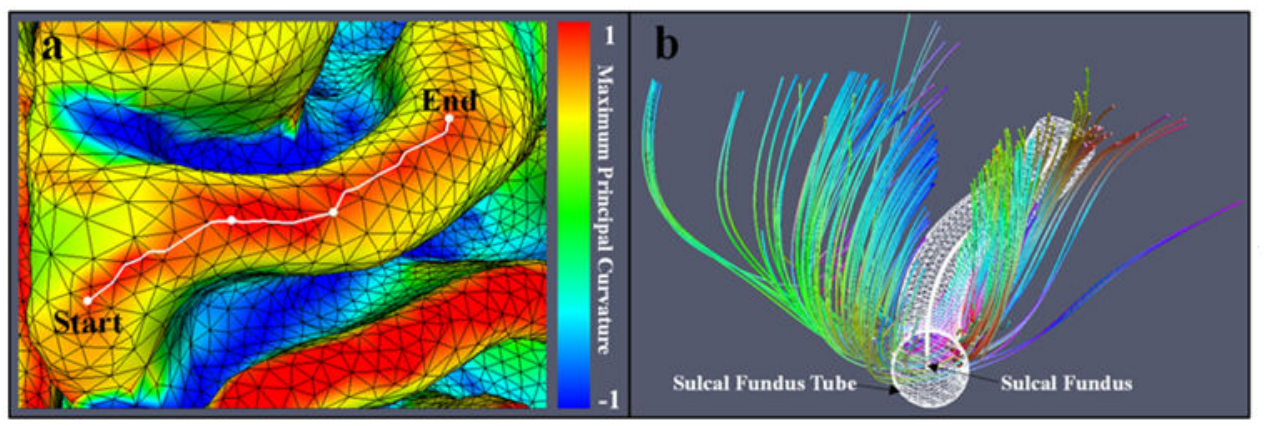

Figure 4.

(a) Illustration of extracting gyral crest line on cortical surface. White dots are manually and sequentially selected from 'Start' to 'End' and the white curve is the geodesic path backtracking from 'End' to 'Start' on the surface, which is color-coded according to the maximum principal curvature bar on the right side. (b) Illustration of extracting U-shape fibers from the sulcal fundus. White curve is the sulcal fundus, and the white mesh frame is a $3 \mathrm{~mm}$ radius tube of the sulcal fundus. Color curves are U-shape fiber bundle passing through the tube. 


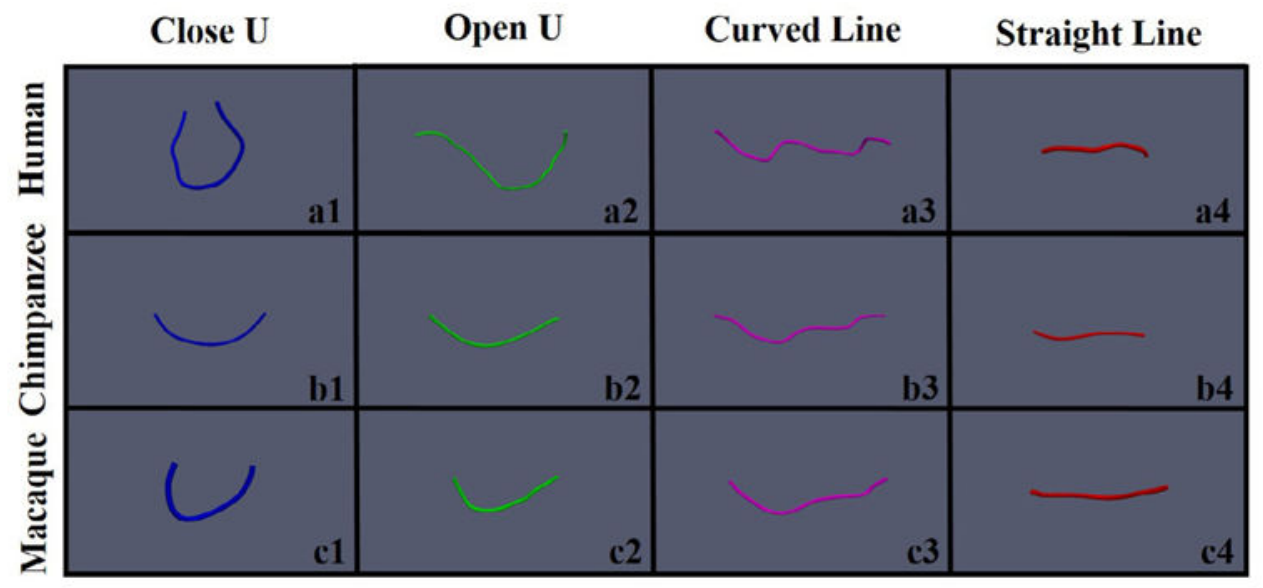

Figure 5.

Comparison of cluster centers obtained by separately applying AP algorithm on human DTI dataset, chimpanzee DTI dataset and macaque DTI dataset. 


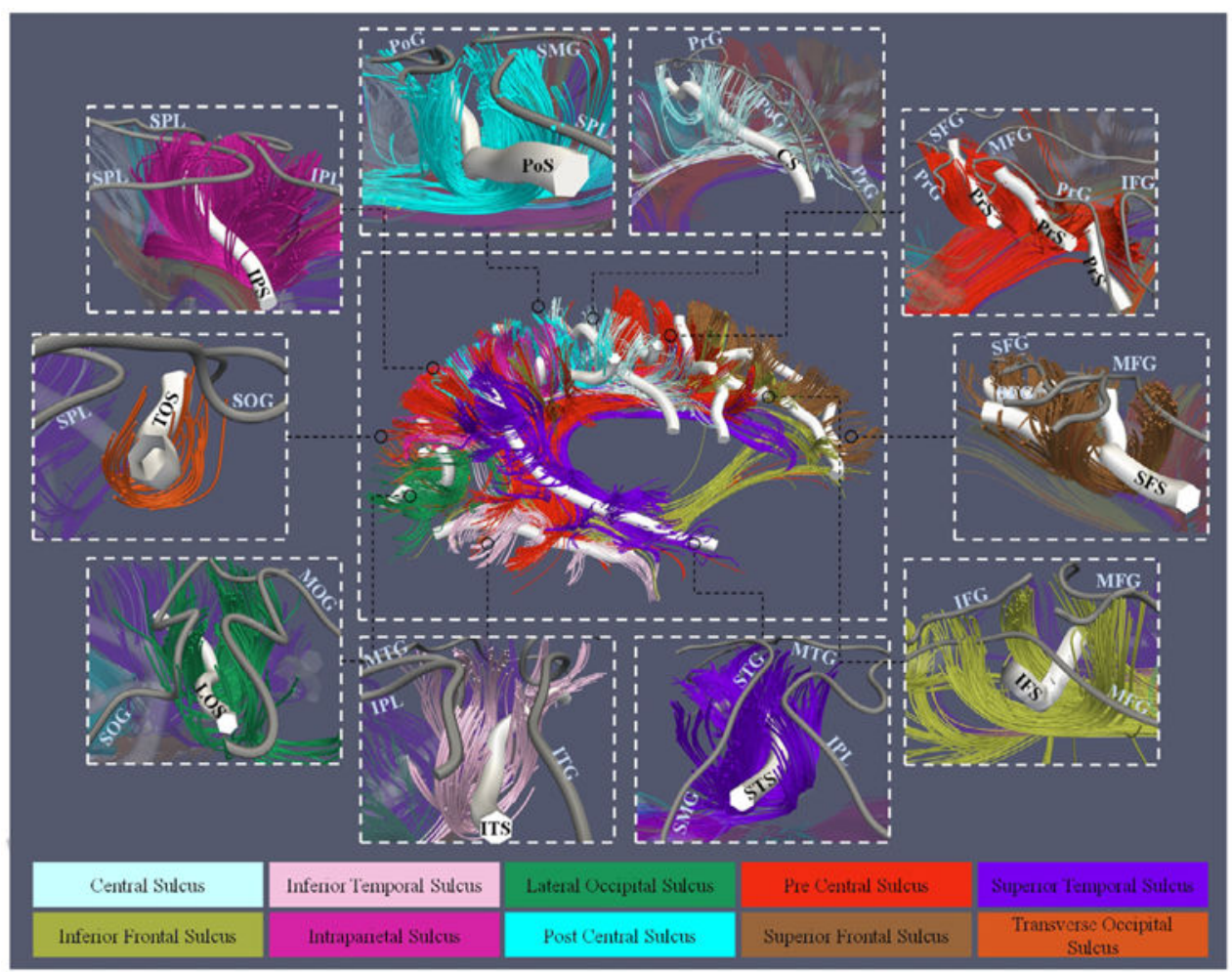

Figure 6.

Joint representation that U-shape fibers course around sulci and connect neighboring gyri on the left hemisphere of human DSI data. Fibers which course around the same sulcus are shown with the same color according to the color table. White tubes are the sulcal fundi and gray tubes are the gyral crest lines. The radius of sulcal fundi tubes is $2 \mathrm{~mm}$ and the radius of gyral crest line tubes is $0.5 \mathrm{~mm}$. The sulcus names are annotated on the sulcal tubes with black color and the gyrus names are annotated beside them with white color. 


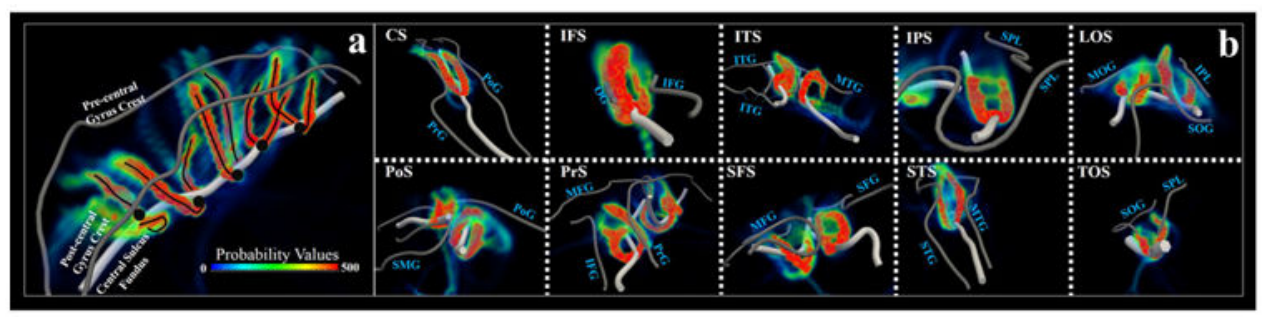

Figure 7.

(a) Joint representation that U-shape fibers course around sulci and connect neighboring gyri on the left hemisphere in the HCP data. White tube: central sulcus fundus. Gray tubes: precentral/post-central gyrus crests. Black dots: seed points selected along the sulcus fundus. Black curves represent the skeleton lines of pathways with high probabilistic values; (b) Joint representation that U-shape fibers course around sulci and connect neighboring gyri on the left hemisphere in the HCP data. 


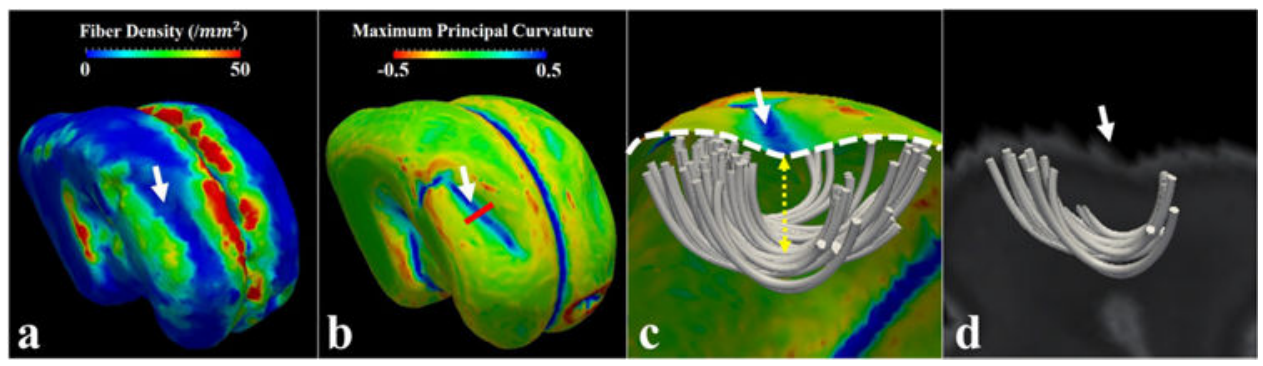

Figure 8.

(a) Fiber end density mapped onto gray matter surface of a fetus brain; (b) The maximum principal curvature map of the gray matter surface; (c) A joint visualization of fibers (white tubes) and a cross-section (white dashed curve) of the surface at the position of red solid line in (b). Yellow dashed arrow indicates the distance between U-shape fiber bottoms and sulcal fundi. (d) T1-weighted MRI cross-section image taken at the same position as (c) is used as background. The white arrow highlights a sulcus. 

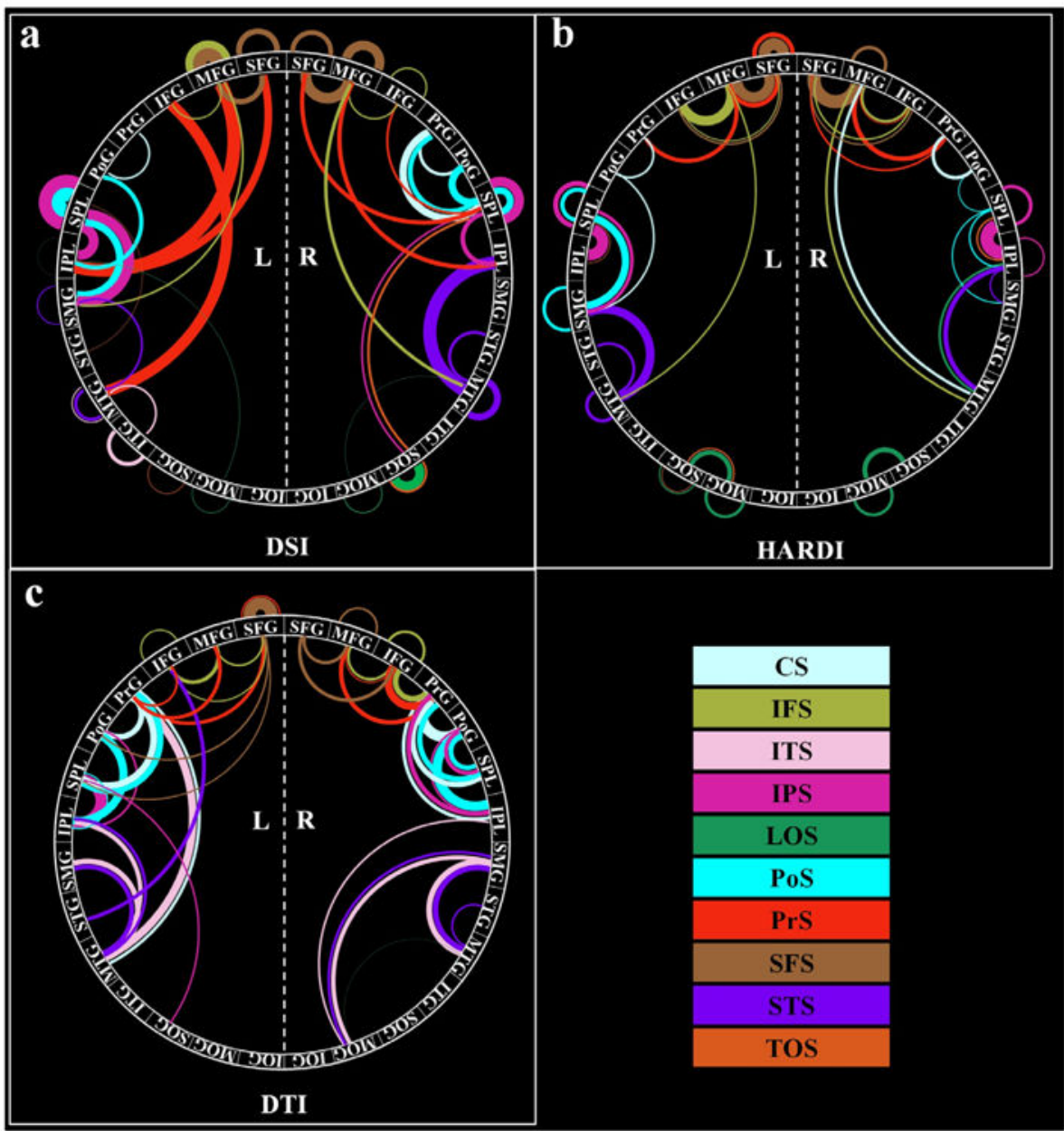

Figure 9.

The U-shape streamline fiber connectivity map of human data: (a) DSI; (b) HARDI; (c) DTI. Note that the connectivities preserved in each sulcus are the top three ones in terms of strength. 


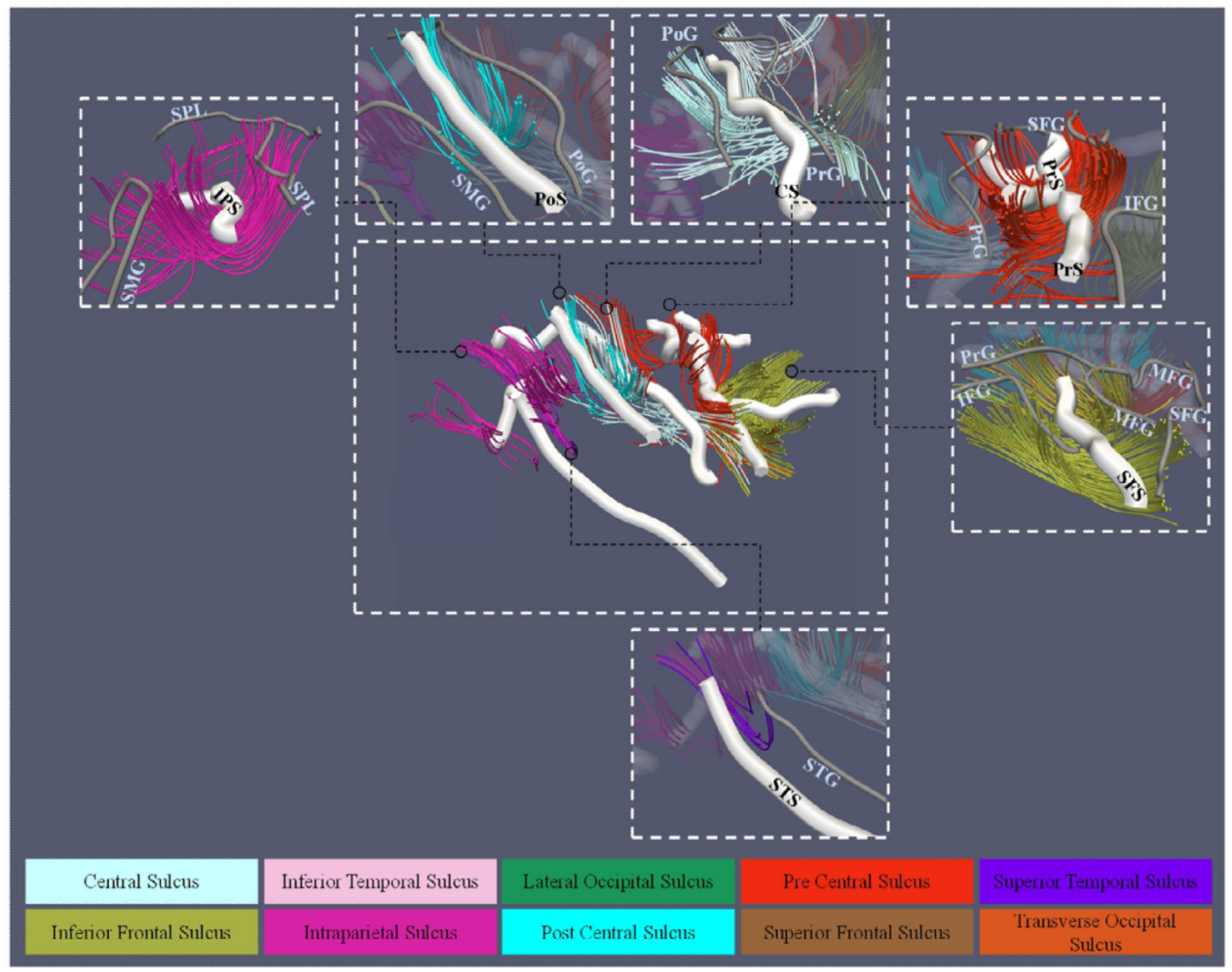

Figure 10.

Joint representation that U-shape fibers course around sulci and connect neighboring gyri on the left hemisphere in chimpanzee DTI data. Fibers which course around the same sulcus are shown with the same color according to the color table. 


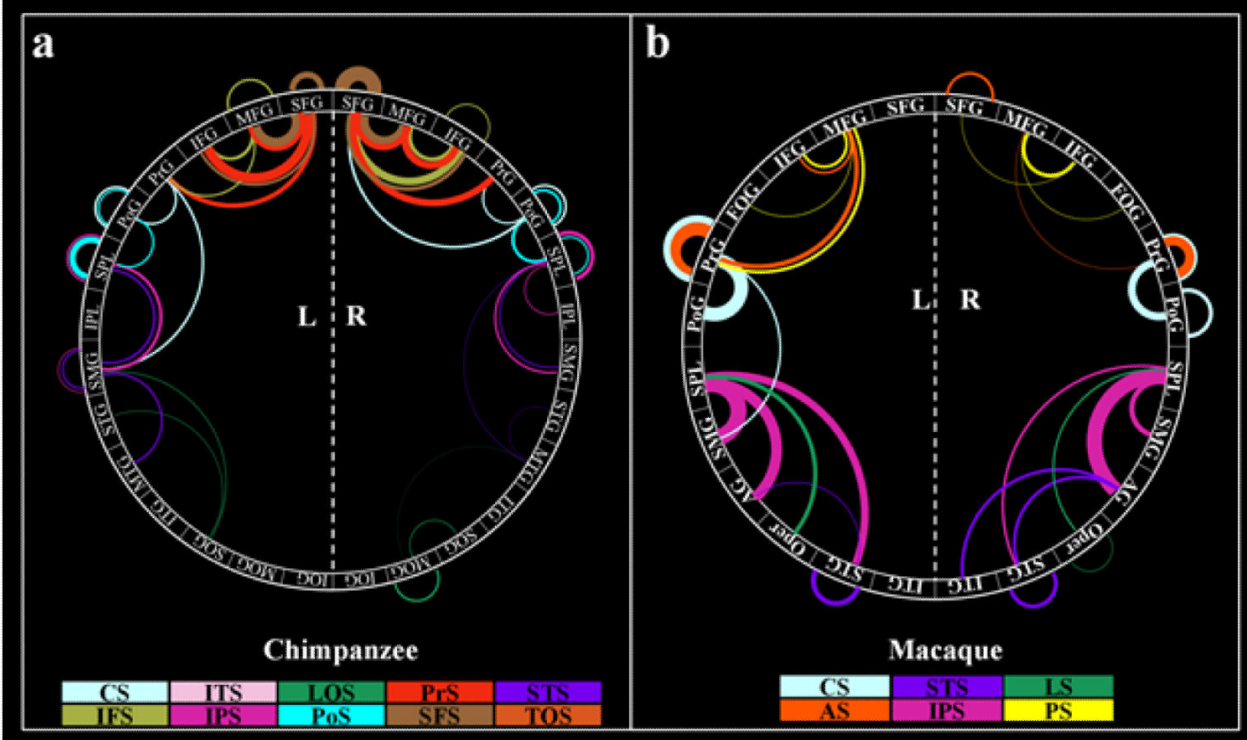

Figure 11.

The U-shape fiber connectivity map in (a) chimpanzee DTI data and (b) macaque DTI data. 


\begin{tabular}{|c|c|c|c|c|c|c|c|c|c|c|}
\hline & $\begin{array}{l}\overrightarrow{0} \\
\frac{50}{\approx z}\end{array}$ & 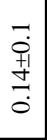 & $\mid \begin{array}{c}0 \\
0 \\
0 \\
0 \\
0 \\
0 \\
0\end{array}$ & $\mid \begin{array}{c}0 \\
0 \\
+1 \\
+1 \\
0 \\
0\end{array}$ & $\begin{array}{l}7 \\
0 \\
01 \\
0 \\
0 \\
0\end{array}$ & 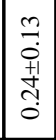 & $\mid \begin{array}{l}0 \\
0 \\
0 \\
+1 \\
0 \\
0 \\
0\end{array}$ & 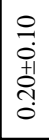 & $\begin{array}{l}= \\
0 \\
\stackrel{+}{1} \\
\stackrel{1}{0}\end{array}$ & \\
\hline & 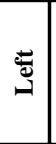 & 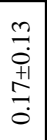 & $\mid \begin{array}{l}= \\
0 \\
01 \\
0 \\
0 \\
0\end{array}$ & $\mid \begin{array}{l}0 \\
0 \\
01 \\
+1 \\
0 \\
0\end{array}$ & 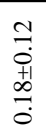 & 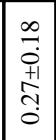 & \begin{tabular}{|l|}
$\infty$ \\
0 \\
0 \\
+1 \\
0 \\
0 \\
0
\end{tabular} & \begin{tabular}{l} 
\pm \\
$\dot{0}$ \\
+1 \\
\multirow{1}{1}{} \\
0
\end{tabular} & $\begin{array}{l}+ \\
0 \\
0 \\
0+1 \\
0 \\
0 \\
0\end{array}$ & \\
\hline$\overline{\underline{\underline{x}}}$ & $\underset{\substack{\overrightarrow{0} \\
: \overrightarrow{0}}}{\approx}$ & 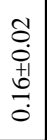 & 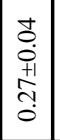 & $\mid \begin{array}{c}0 \\
0 \\
+1 \\
\stackrel{+1}{C} \\
0 \\
0\end{array}$ & 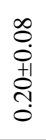 & 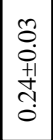 & 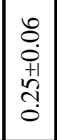 & $\begin{array}{l}0 \\
0 \\
0 \\
0 \\
0 \\
0 \\
0\end{array}$ & 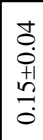 & \\
\hline 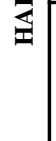 & 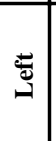 & $\begin{array}{l}\overrightarrow{0} \\
\dot{0} \\
\stackrel{1}{0} \\
\stackrel{0}{0}\end{array}$ & 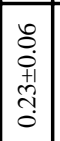 & 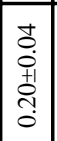 & $\begin{array}{l}\text { Oे } \\
\text { +1 } \\
\stackrel{1}{2} \\
\stackrel{0}{0}\end{array}$ & 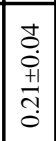 & 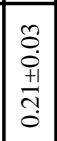 & $\mid \begin{array}{l}\overline{0} \\
\dot{0} \\
+1 \\
\stackrel{+1}{0} \\
0\end{array}$ & $\begin{array}{l}0 \\
0 \\
+1 \\
+1 \\
-1 \\
0\end{array}$ & \\
\hline & 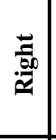 & $\begin{array}{l}\overline{0} \\
\dot{0} \\
\text { गे } \\
0 \\
0\end{array}$ & $\begin{array}{l}0 \\
0 \\
0 \\
0 \\
0 \\
0\end{array}$ & $\mid \begin{array}{c}0 \\
0 \\
\frac{\dot{p}}{2} \\
\stackrel{0}{0}\end{array}$ & \begin{tabular}{|l}
$\infty$ \\
0 \\
0 \\
†े \\
0 \\
0
\end{tabular} & $\mid \begin{array}{l}0 \\
0 \\
\dot{0} \\
+1 \\
0 \\
0 \\
0\end{array}$ & 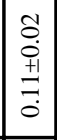 & $\mid \begin{array}{l}0 \\
0 \\
\dot{1} \\
0 \\
0 \\
0 \\
0\end{array}$ & $\begin{array}{l}m \\
0 \\
+1 \\
\stackrel{+1}{0} \\
0\end{array}$ & \\
\hline & $\Phi$ & $\begin{array}{l}\text { o. } \\
\dot{\dagger} \\
0 \\
0 \\
0\end{array}$ & $\mid \begin{array}{c}0 \\
0 \\
0 \\
\frac{+1}{3} \\
0 \\
0\end{array}$ & $\begin{array}{l}0 \\
0 \\
0 \\
\hat{1} \\
\hat{i} \\
0\end{array}$ & 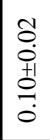 & \begin{tabular}{|l|l} 
\\
0 \\
$\dot{0}$ \\
0 \\
0 \\
0 \\
0 \\
0
\end{tabular} & 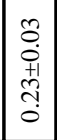 & $\mid \begin{array}{l}\overline{0} \\
\dot{0} \\
+1 \\
\stackrel{+1}{0} \\
\end{array}$ & $\begin{array}{l}0 \\
0 \\
0 \\
0 \\
0 \\
0 \\
0\end{array}$ & \\
\hline$\frac{\mathscr{n}}{\bar{g}}$ & & U & 里 & $\cong$ & $\therefore$ & $\begin{array}{l}n \\
2\end{array}$ & 怘 & 5 & \% & \\
\hline
\end{tabular}

Med Image Anal. Author manuscript; available in PMC 2015 July 01. 


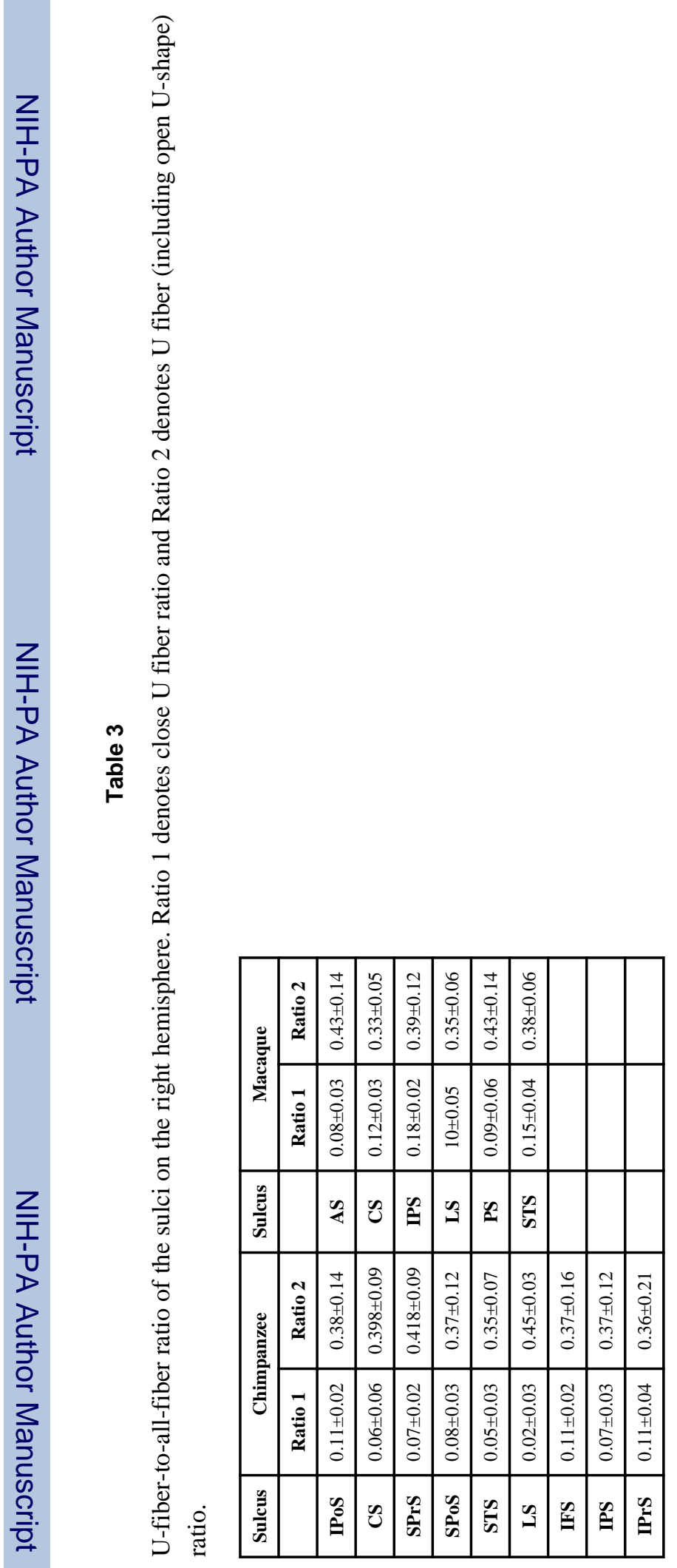

Med Image Anal. Author manuscript; available in PMC 2015 July 01. 


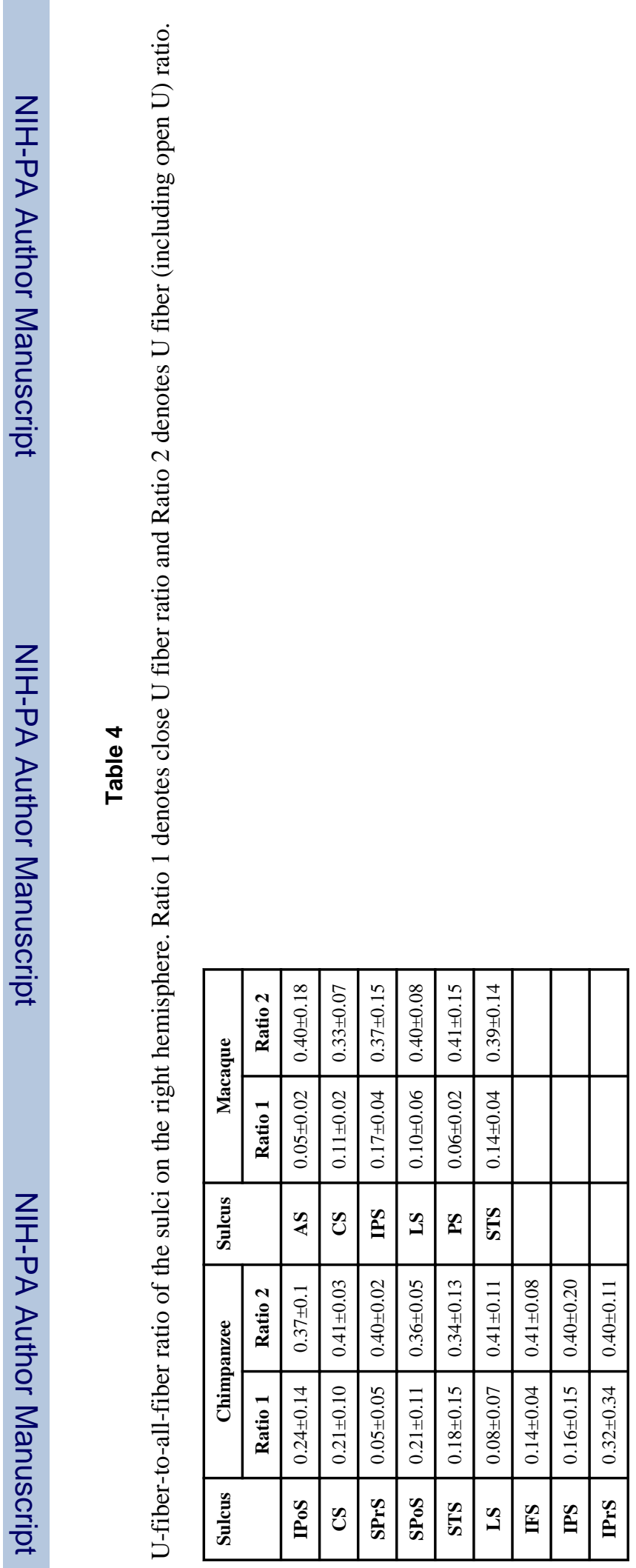

Med Image Anal. Author manuscript; available in PMC 2015 July 01. 